\title{
The Dynamics of a Periodically Forced Cortical Microcircuit, With an Application to Schizophrenia*
}

\author{
Dorea Vierling-Claassen ${ }^{\dagger}$ and Nancy Kopell ${ }^{\ddagger}$
}

\begin{abstract}
Synchronous neural activity in the brain in the "gamma" and "beta" frequency bands $(15-70 \mathrm{~Hz})$ is thought to be important for sensory processing and is altered in schizophrenia. In a previous study, gamma/beta click-train auditory stimuli were used to probe cortical oscillatory activity in control and schizophrenic subjects. We found that control subjects exhibited preferential $40 \mathrm{~Hz}$ responses to both 20 and $40 \mathrm{~Hz}$ stimulations, while schizophrenic subjects had enhanced $20 \mathrm{~Hz}$ responses to the same stimuli [D. Vierling-Claassen, P. Siekmeier, S. Stufflebeam, and N. Kopell, J. Neurophysiol., 99 (2008), pp. 2656-2671]. High-dimensional computational network models constructed previously, which were based on evidence of altered inhibition in schizophrenia, numerically generated the entrainment behaviors observed experimentally. However, questions regarding the dynamic origin of model behaviors remained. It was not clear that the $20 \mathrm{~Hz}$ response to $40 \mathrm{~Hz}$ drive in the schizophrenic network was robust to parameter changes, which would be necessary for the predicted mechanism to explain data from a heterogeneous subject population. In the schizophrenic network we observed $30 \mathrm{~Hz}$ drive responses with a frequency component below $30 \mathrm{~Hz}$, for which no analogue appeared in experimental data, and wondered if these were dynamically distinct from the modeled $20 \mathrm{~Hz}$ response to $40 \mathrm{~Hz}$ drive. We also wished to explore the role of background noise in model behavior. To address these questions, we consider a system of two mutually coupled oscillators representative of neural cells, driven periodically in the gamma/beta frequency band. We show that there is a one-parameter family of discontinuous discrete maps, whose dynamics clarifies issues of robustness, classifies entrainment patterns, and provides insight into the role of excitatory noise.
\end{abstract}

Key words. discrete map, schizophrenia, inhibition, excitation, periodic drive, forced oscillator, cortex, auditory, gamma, beta

AMS subject classifications. 34C15, 37E99, 37N25, 92C20

DOI. $10.1137 / 080738052$

1. Introduction. In the nervous system, the $40 \mathrm{~Hz}$ "gamma" and $20 \mathrm{~Hz}$ "beta" rhythms are thought to be important for early sensory processing $[2,12,17]$. There is also evidence that gamma rhythms break down in the auditory system in schizophrenia $[20,22,28]$. The current work is motivated by a previous experimental and computational study that used click-train stimuli to compare gamma and beta band oscillatory activity in auditory cortex in control and schizophrenic subjects. This work, reviewed more extensively in the discussion, showed roughly that control subjects had an increased $40 \mathrm{~Hz}$ response to both $20 \mathrm{~Hz}$ and $40 \mathrm{~Hz}$ auditory stimulation, while schizophrenic subjects had a preference for $20 \mathrm{~Hz}$ response

\footnotetext{
* Received by the editors October 14, 2008; accepted for publication (in revised form) by P. Holmes March 9, 2009; published electronically June 3, 2009. This work was supported by NSF Award DMS-0211505 and Burroughs Wellcome Fund Award 1001749.

http://www.siam.org/journals/siads/8-2/73805.html

${ }^{\dagger}$ Athinoula A. Martinos Center for Biomedical Imaging, Department of Radiology, Massachusetts General Hospital, 149 13th Street, Charlestown, MA 02129 (dorea@nmr.mgh.harvard.edu).

${ }^{\ddagger}$ Department of Mathematics and Statistics, Center for BioDynamics, Boston University, 111 Cummington St., Boston, MA 02215 (nk@math.bu.edu).
} 
to the same frequencies [28].

The computational models constructed for this work were based on evidence of weakened, and more importantly, temporally extended neural inhibition in schizophrenia [21]; included both excitatory and inhibitory cells; and numerically generated the observed auditory entrainment behavior. These numerical simulations showed "beat skipping" of the excitatory cells relative to the $40 \mathrm{~Hz}$ click input in the schizophrenic network, which generated the $20 \mathrm{~Hz}$ response, due to the presence of extended inhibition. However, several questions regarding the dynamic origin of the different behaviors, in particular with regard to the beat skipping, remained to be examined. It was not clear how robust this beat skipping response was, or how dependent on fine-tuning of other network parameters. If the mechanism we proposed was correct, the behavior would need to be extremely robust in order to be detectable in noisy data from a highly heterogeneous patient population. In addition, while tuning the model, particularly in the schizophrenic case with $30 \mathrm{~Hz}$ drive, we observed several alternate entrainment patterns with less frequent skipping (for example, failing to respond to every third drive input). No analogue for these behaviors appeared in the experimental data; thus we wished to establish whether or not the desired beat skipping behavior was truly dynamically distinct from these alternate entrainment patterns. We also found that the model was somewhat dependent on excitatory background noise, particularly in the schizophrenic case with $30 \mathrm{~Hz}$ drive, and it was not clear why this was so.

To address these questions we work in a more general analytical setting. We consider a periodically driven system of two mutually coupled oscillators representative of neural cells, one excitatory (E) and one inhibitory (I). The I cell inhibits both itself and the E cell, and the $\mathrm{E}$ cell receives periodic excitatory drive in the gamma and beta bands. We investigate how the pattern of oscillator entrainment to the drive depends on the time-course of inhibition. We show that there is a one-parameter family of discontinuous discrete maps, whose dynamics clarifies the issues of robustness described above for the schizophrenic case, as well as explaining much of the control entrainment behavior and the variety of entrainment patterns observed, and for certain cases clarifies the importance of excitatory noise in the network model.

We will consider network behavior in the cognitively important gamma and beta bands of neural activity. Progress has been made in understanding the role of inhibition in maintaining synchrony $[1,25]$, and in understanding the mechanisms and functional importance of gamma rhythms in networks that contain both excitation and inhibition $[3,5,6,7,11,23]$. Though this work is motivated by a specific neuroscience application, the associated method has far wider relevance. Unlike the larger literature that deals with forced oscillators [16], we describe a forced circuit in which the circuit has internal degrees of freedom that are influenced by the forcing. Thus the work represents a new methodological direction.

2. Model system and parameters. As in previous computational studies of gamma/betarange behavior in reduced cortical networks $[4,5]$, we implement our cortical microcircuit using the "theta" model, in which a spiking cell is represented by a point traveling around a circle with varying speed. Time is considered on the scale of milliseconds, as this is the relevant timescale for consideration of neuronal spiking. Use of this simplified model, in which each cell is governed by only three dynamic variables (one for the cell and two controlling synaptic input), allows us to use intuitive techniques of qualitative dynamic analysis, while

Copyright (c) by SIAM. Unauthorized reproduction of this article is prohibited. 
still permitting focus on the most important parameters underlying the motivation for this work: the frequency of periodic drive and the time-course of inhibition.

We consider two cells, each governed by the so-called theta equation

$$
\theta^{\prime}=1-\cos \theta+B(1+\cos \theta) .
$$

This model is closely related to the quadratic integrate-and-fire model [4] and Type I HodgkinHuxley [14] analysis, and $\theta$ is the voltage variable, transformed, while parameter $B$ is the transformation of the applied current. When $\theta=\pi$, we say that the cell fires.

When $B<0$, there exist two fixed points at $\theta_{ \pm}= \pm \cos ^{-1}\left(\frac{1+B}{1-B}\right)$. The negative fixed point is stable; the positive fixed point is unstable, and the cell is silent. As $B$ is increased, the system undergoes a saddle node bifurcation, and there are no fixed points for $B>0$. For positive $B$ the cell will fire repeatedly at a fixed period, moving more slowly near $\theta=0$ and rapidly near $\theta=\pi$.

In our case, $B$ is time dependent, with

$$
B=b+g_{E}-g_{I},
$$

where $g_{E}$ and $g_{I}$ are excitatory and inhibitory inputs, respectively, each governed by a decaying exponential equation:

$$
\begin{gathered}
g_{E}^{\prime}=-\tau_{E} g_{E}, \\
g_{I}^{\prime}=-\tau_{I} g_{I} .
\end{gathered}
$$

In the equations for $\theta_{E}$ and $\theta_{I}$ we will distinguish between variables governing excitation onto $\theta_{E}\left(g_{E E}\right)$ and excitation onto $\theta_{I}\left(g_{E I}\right)$, and similarly for inhibition $\left(g_{I E}\right.$ and $\left.g_{I I}\right)$. Although the equations for excitation and inhibition are identical between the cells (e.g., the equation (2.3) governing $g_{E I}$ is identical to that governing $g_{E E}$ ), the variables are nonetheless dynamically distinct because they are subject to reset conditions that depend on network activity. Thus, the full set of equations is (2.3), (2.4), and

$$
\begin{gathered}
\theta_{E}^{\prime}=1-\cos \theta_{E}+\left(b+g_{E E}-g_{I E}\right)\left(1+\cos \theta_{E}\right), \\
\theta_{I}^{\prime}=1-\cos \theta_{I}+\left(b+g_{E I}-g_{I I}\right)\left(1+\cos \theta_{I}\right) .
\end{gathered}
$$

The full system has six dimensions (four synaptic variables and two cellular variables). Figure 1 shows network connectivity and the role of each variable.

Synaptic activity is modeled by instantaneous positive resets of the four synaptic variables. Each time the E cell receives periodic drive (at times $n T$ for $n$ a positive integer), the variable $g_{E E}$, which decays exponentially between inputs (as in (2.3)), is reset to the constant $k_{E E}$ (as shown in Figure 2). The reset of $g_{E E}$ directly models excitatory synaptic input by pushing the cell towards the spiking regime, where $B>0$. In addition, when periodic excitation arrives, the variable $\theta_{E}$ is shifted positively by $S$ radians.

This positive shift in $\theta_{E}$ is needed to account for a difference between the continuous synapses used in the previous larger network model [28] and the simplified synapses with an instantaneous reset used in this two-cell case. With the continuously defined synapse in the

Copyright (c) by SIAM. Unauthorized reproduction of this article is prohibited. 


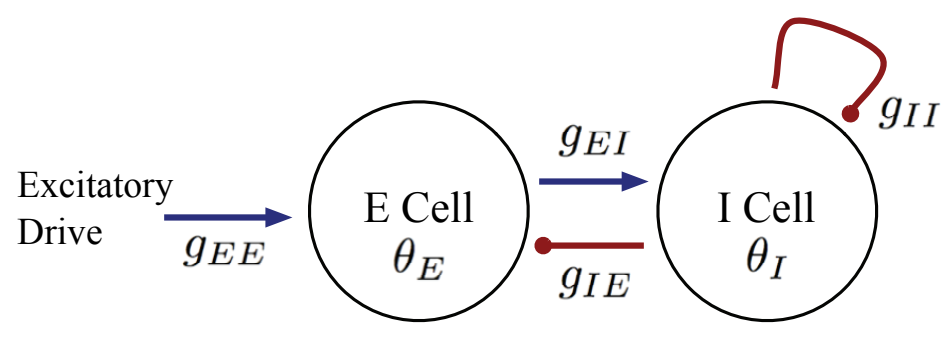

Figure 1. Figure showing network connectivity and relationships between the six model dimensions. See (2.3)-(2.6). Excitatory synapses are shown in blue with pointed arrow heads. Inhibitory synapses are shown in red with rounded arrow heads.

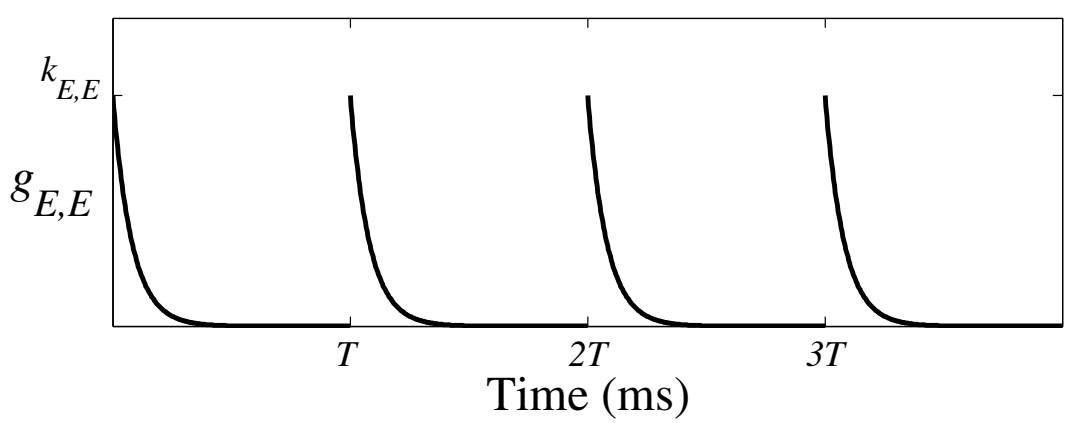

Figure 2. Figure showing values of variable $g_{E E}$ in time (excitatory synaptic input to the $E$ cell). The value of $g_{E E}$ is reset to $k_{E E}$ with period $T$, and decays exponentially at rate $\tau_{E}$ between resets.

full system, the synaptic variables had a rapid rise time, and when the synapse was excitatory, the $\theta$ variable changed a small amount in the positive direction during the rapid rise, as the solution traveled quickly through a region of phase space where $\theta^{\prime}>0$. In this reduced system, however, to facilitate further reduction to a map, we discretize the synaptic rise. In order to maintain agreement between the simplified system considered here and the large continuous network studied previously, when excitation is input into either cell, both the relevant $g_{E}$ variables $\theta$ are reset. However, when a cell receives inhibition, only the relevant $g_{I}$ variable is reset, with no corresponding shift in $\theta$, because with the continuous synapses, the minimal shift in $\theta$ due to the inhibitory rise time does not cause any discrepancy in behavior between the previous larger network and this two-cell simplification. The reset in $\theta$ with excitation is not essential for derivation of the map but is necessary to maintain agreement between the previous data-driven network and the simplified case considered here.

If the E cell fires as a result of an excitatory input, then excitation is provided to the I cell by way of a reset of $g_{E I}$ to $k_{E I}$ and a positive shift in $\theta_{I}$ by $S$ radians. If the inhibitory spikes as a result of this input, then $g_{I E}$ is reset to $k_{I E}$, and simultaneously $g_{I I}$ is reset to $k_{I I}$, where the latter corresponds to inhibition provided from the I cell to itself. No reset in $\theta_{E}$ or $\theta_{I}$ takes place when the inhibitory synaptic variables are reset.

Though we consider a specific set of parameters here, the underlying structure of the map that we derive remains applicable, provided we make the following assumptions about the 
Table 1

Parameter values used for simulations, unless otherwise stated.

\begin{tabular}{|c|c|l|}
\hline Parameter & Value & Network function \\
\hline \hline$\tau_{E}$ & $1 / 2$ & Excitatory decay \\
\hline$\tau_{I}$ & $1 / 8$ (control) & Inhibitory decay \\
\hline$k_{E E}$ & $1 / 28$ (schizophrenic) & \\
$k_{E I}$ & 0.3 & Strength of excitatory drive \\
$k_{I E}$ & 0.5 & Strength of excitation from E to I cell \\
$k_{I I}$ & 0.15 & Strength of inhibition from I to E cell \\
\hline$b$ & 0.2 & Strength of inhibition from I cell to itself \\
\hline$S$ & -0.01 & Ensures cells are silent in absence of input \\
\hline$T$ & 0.285 radians & Reset in $\theta$ with excitation \\
\hline
\end{tabular}

system (all of which are satisfied by the specific parameter regime that we consider).

Assumption 2.1. Both cells are silent in the absence of excitatory input.

Assumption 2.2. Parameters are such that a spike in the E cell guarantees a spike in the I cell (i.e., the reset value $k_{E I}$ is large enough to ensure spiking of the I cell).

Assumption 2.3. Both the drive input to the E cell and the excitatory input to the I cell are such that only a single spike results from a single input (i.e., reset values $k_{E I}$ and $k_{E E}$ are not so large that multiple spikes occur per excitatory input).

Assumption 2.4. The balance between excitatory decay time $\tau_{E}$ and period $T$ is such that the value of excitation in both the $E$ cell and the I cell is negligible at times $n T^{-}$. That is, we assume that $T$ is long enough and excitatory decay is fast enough that solutions are very near the $g_{E}=0$ plane just prior to excitatory input from the drive.

Assumptions 2.1, 2.2, and 2.3 are necessary for the discrete map to be well defined. Assumption 2.4 is an approximation that permits localization of solutions prior to periodic input, which permits derivation of the map. The impact of this assumption, as well as the related Assumption 5.1 below, is discussed in section 8 .

The specific parameters that we use here are taken directly from our previous work [28] and are shown in Table 1 . We take $\tau_{E}=1 / 2$, which is representative of fast (1-4 ms) exponential decay of $\alpha$-amino-3-hydroxy-5-methyl-4-isoxazolepropionic acid (AMPA)-type excitation in cortical cells $[18,30]$. The control inhibitory decay rate $\tau_{I}=1 / 8$ (8 ms decay) is selected to be representative of gamma-aminobutyric acid, type $\mathrm{A}\left(\mathrm{GABA}_{A}\right)$ decay kinetics $[26,27,29]$. There is some debate about the biophysically relevant decay times for $\mathrm{GABA}_{A}$ in cortex, with both fairly short (3.71 ms) and extremely long (33.2 ms) decay times reported [15, 19], though there is agreement that $\mathrm{GABA}_{A}$ decay is generally longer than AMPA-type excitatory currents. We choose $8 \mathrm{~ms}$ decay both because it is an intermediate value in the range of reported values and because decay times in the 6 to $10 \mathrm{~ms}$ range are traditionally used in the modeling literature $[4,5,8,29]$. We believe that what is of primary importance for the dynamic mechanisms presented here is that inhibitory decay be longer than excitatory decay in the control, for which there is experimental agreement.

We also consider a longer inhibitory decay $\tau_{I}=1 / 28$, which models a possible extension of inhibitory decay in schizophrenic cortical networks due to a reduction in the available GABA transporter, GAT-1 [21]. Although there is evidence of extended decay times due to

Copyright $\odot$ by SIAM. Unauthorized reproduction of this article is prohibited. 
reduced GAT-1 [24], it is impossible to infer biophysically reasonable values for an extended decay time, so this parameter is selected to generate the desired network behavior. As will be discussed below, the model is not dependent on the exact selection of this parameter, but rather the desired model behavior is present for a wide range of possible decay times.

In previous research with a larger network [28], strength of synaptic activity (here represented by the constants $k_{E E}, k_{E I}, k_{I E}$, and $k_{I I}$ controlling synaptic resets) was selected to generate the control entrainment behavior. That is, with inhibitory decay rate $\tau_{I}=1 / 8$, synaptic strength parameters were tuned to generate entrainment to 30 and $40 \mathrm{~Hz}$ drive and a mixed-mode response to $20 \mathrm{~Hz}$ drive containing both 20 and $40 \mathrm{~Hz}$ components (where the $40 \mathrm{~Hz}$ component depends on excitatory background noise not included in this reduced model). The values of the synaptic strength parameters used in this two-cell network are taken directly from the previous work, and are shown in Table 1.

3. Model behavior and motivating questions. Figures 3 and 4 show the behavior of the two-cell network ((2.3)-(2.6), with synaptic resets described in section 2$)$. With $\tau_{I}=1 / 8$ (control case), the system entrains in a one-to-one fashion to drives of 20,30 , and $40 \mathrm{~Hz}$, which correspond to $T=50,33.33$, and 25, respectively. For slower decay of $\tau_{I}=1 / 28$ (schizophrenic case), the network either entrains directly to the drive frequency, responding to each drive input, or fails repeatedly to respond to some drive pulses in a fixed manner. For period $T=50 \mathrm{~ms}$ ( $20 \mathrm{~Hz}$ drive), the network responds to every input from the drive (Figure $4(\mathrm{C})$ ). When the period is shortened to $T=33.33 \mathrm{~ms}(30 \mathrm{~Hz})$, the network fails to respond to every third input (Figure 4(B)). For an even shorter period of $T=25 \mathrm{~ms}(40 \mathrm{~Hz})$, the network fails to respond to alternate drive inputs (Figure 4(A)). It is the relationship between the period of the drive and the time-course of inhibition that controls what form of skipping behavior the network displays.

Table 2 summarizes the entrainment behaviors observed experimentally, in our computational model [28] and in this two-cell reduction. Figure 2 in [28] shows experimental behavior of the auditory entrainment experiment, and Figures 4 and 5 in [28] show the behavior of the full computational model. Note that the previous study also contains a much more detailed biophysical model (called the GENESIS model) that we do not directly consider here. The experimental results and full model with noise (columns 3 and 4 in Table 2) show identical frequency content, as do the full model without noise and the two-cell model (columns 5 and 6 of Table 2).

However, the three behaviors labeled "Noise dependent" in the table are not identical in the simplified two-cell model. For these three behaviors, noise in the network provides a background level of excitation that is then controlled by the deterministic network dynamics. We will not discuss in detail the noise dependencies for 20 and $40 \mathrm{~Hz}$ drive, which are considered in more detail in the previous work. We focus instead on the noise dependence for $30 \mathrm{~Hz}$ drive with long inhibition (next to last row of Table 2), in which the two-cell network skips every third input but the full model entrains.

Our previous study in larger networks left several questions unanswered regarding the schizophrenic case of slow inhibitory decay $\left(\tau_{I}=1 / 28\right)$. In particular, work with the simplified two-cell model can address three questions that could not be satisfactorily answered in the previous larger computational context:

Copyright (c) by SIAM. Unauthorized reproduction of this article is prohibited. 
A. Control, $40 \mathrm{~Hz}$ drive

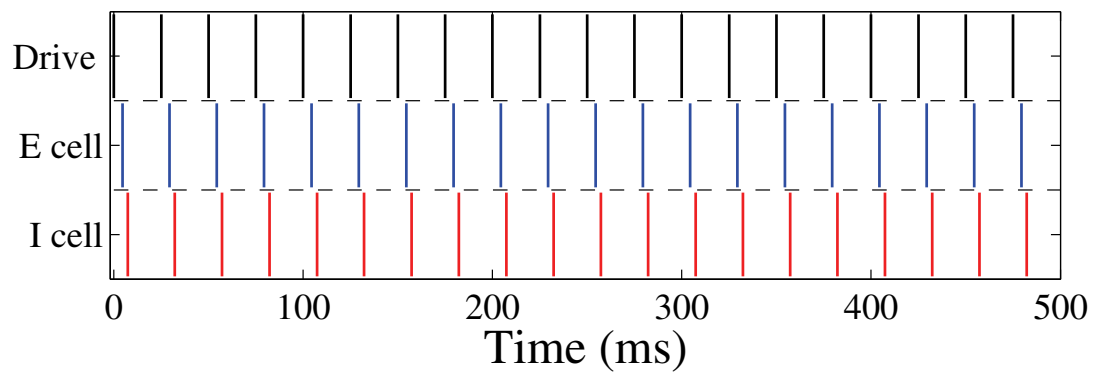

B. Control, $30 \mathrm{~Hz}$ drive

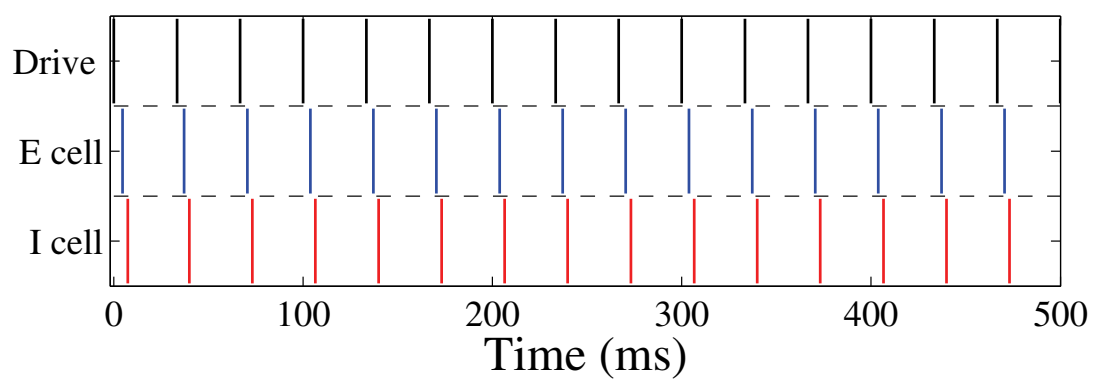

C. Control, $20 \mathrm{~Hz}$ Drive

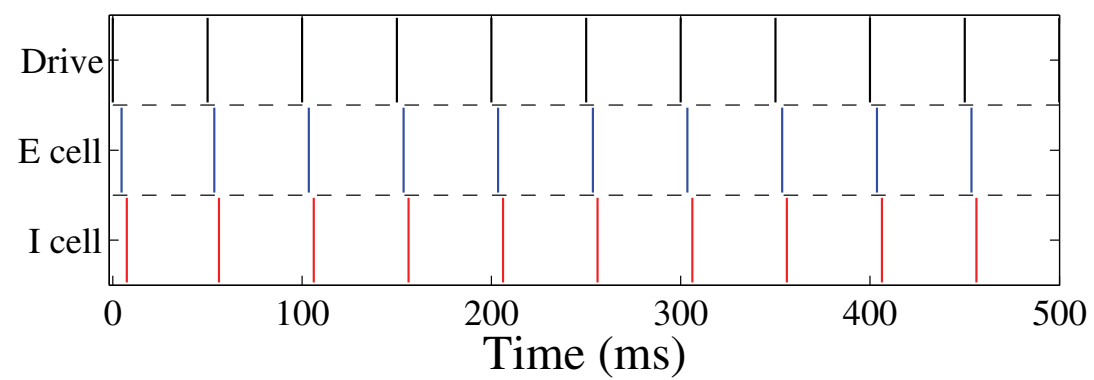

Figure 3. Raster plots for $\tau_{I}=1 / 8$, control case. Parameters as as in Table 1. Vertical lines indicate spike times for excitatory drive (black, top), E cell (blue, middle), and I cell (red, bottom). For the control case with 40, 30, and $20 \mathrm{~Hz}$ excitatory drive (panels $\mathrm{A}, \mathrm{B}$, and $\mathrm{C}$, respectively), both $\mathrm{E}$ and I cells respond to each drive input, and the network entrains to the drive frequency.

1. Is the mechanism that leads to beat skipping robust enough that we should expect to see evidence of this behavior in real data?

2. Less-frequent beat skipping patterns (e.g., skipping every third drive input) were observed in the larger network model, but no evidence for such rhythms was observed experimentally. Are these less-frequent beat skipping patterns dynamically distinct from the alternate beat skipping response?

3. Why is the computational network dependent on the presence of excitatory background noise to generate reliable $30 \mathrm{~Hz}$ entrainment for the schizophrenic case?

Copyright (C) by SIAM. Unauthorized reproduction of this article is prohibited. 
A. Schizophrenic case, $40 \mathrm{~Hz}$ drive

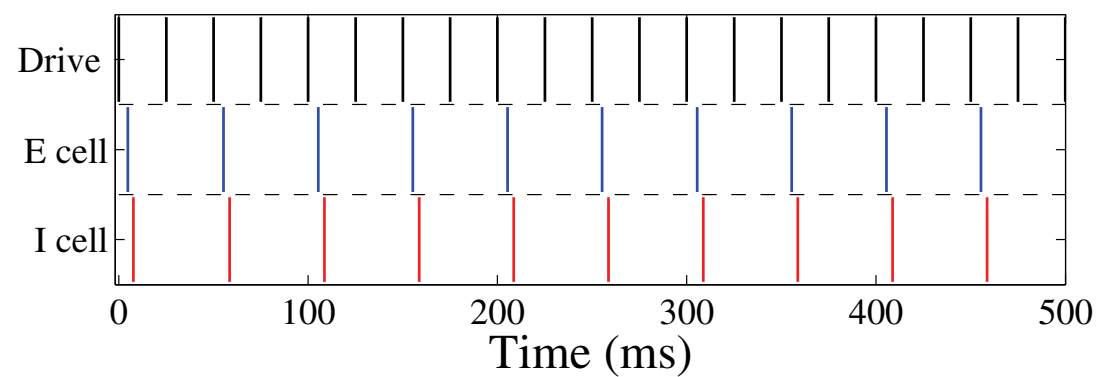

B. Schizophrenic case, $30 \mathrm{~Hz}$ drive

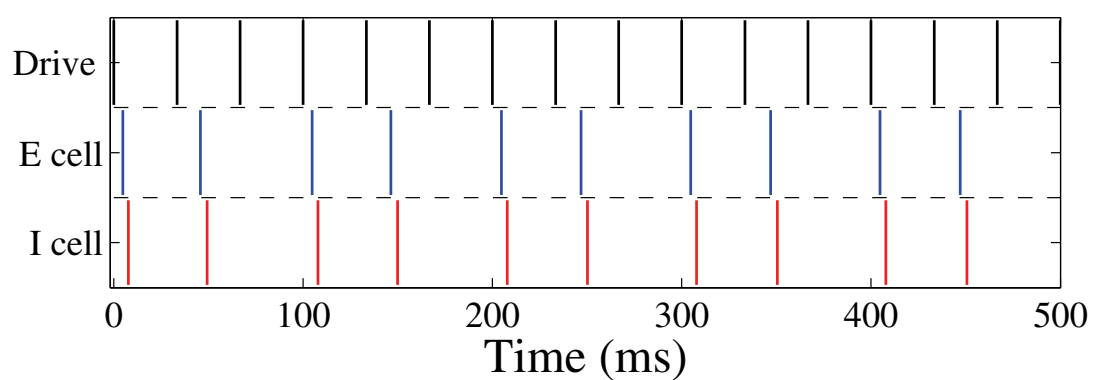

C. Schizophrenic case, $20 \mathrm{~Hz}$ drive

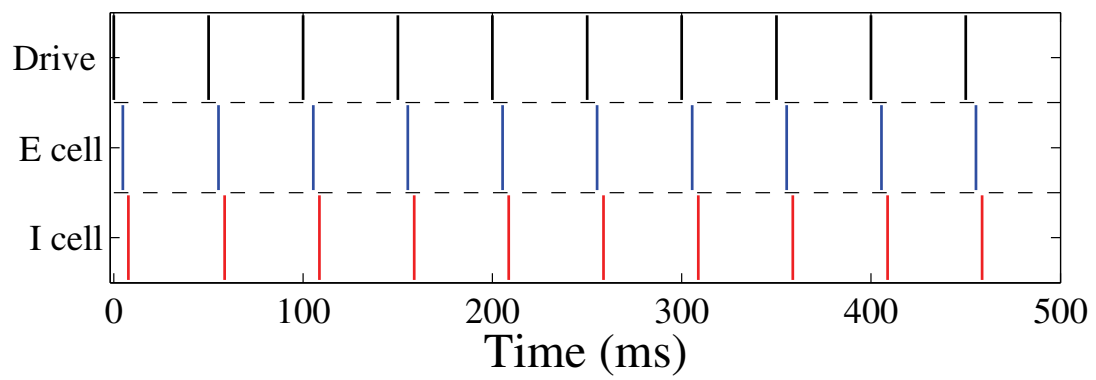

Figure 4. Raster plots for $\tau_{I}=1 / 28$, schizophrenic case. Parameters as as in Table 1. Vertical lines indicate spike times for excitatory drive (black, top), E cell (blue, middle), and I cell (red, bottom). A: With $40 \mathrm{~Hz}$ excitatory drive, the $E$ and I cells respond to alternate drive inputs due to extended inhibition, producing a $20 \mathrm{~Hz}$ network response. B: For $30 \mathrm{~Hz}$ excitatory drive, the $\mathrm{E}$ and I cells fail to respond to every third drive input. This is an example of a sensitive transitional network behavior, and is produced by a dynamic mechanism distinct from that which produces the alternate beat skipping response to $40 \mathrm{~Hz}$ drive. (See section 7.2 for details.) C: Both the E and I cells respond to every drive input at $20 \mathrm{~Hz}$, producing $20 \mathrm{~Hz}$ network output.

In order to answer these questions, we numerically derive a family of discontinuous discrete maps from the reduced ODE system (2.3)-(2.6). Although this family of maps is derived without noise, we are still able to make conclusions about the role of noise in the full computational model, in part because in this simplified context we can clearly identify the mechanisms underlying deterministic behaviors. 
Table 2

Summary of entrainment behaviors, comparing magnetoencephalography (MEG) data, previous full computational model [28], full computational model with noise removed, and the two-cell model described in detail in this paper. Top three rows summarize network behaviors for short inhibition (control case, $\tau_{I}=1 / 8$ ). Bottom three rows summarize network behaviors for extended inhibition (schizophrenic case, $\tau_{I}=1 / 28$ ). In the top and bottom rows, where two frequencies are listed, the experimental data and full computational model produce a mixed-mode response containing both frequencies. However, note that that in both such cases, the $40 \mathrm{~Hz} r e-$ sponse component is noise dependent and is thus not produced by the full model without noise or by the two-cell model. Note also that the $30 \mathrm{~Hz}$ response to $30 \mathrm{~Hz}$ input with long inhibition (second to last row) is also noise dependent and is thus distinct from the behavior of skipping every third input that is present in the full model with noise removed and in the two-cell model.

\begin{tabular}{|c|c|c|c|c|c|c|}
\hline \multicolumn{2}{|c|}{ Network params. } & \multicolumn{4}{|c|}{ Response to drive, $\mathrm{Hz}$} & \\
\hline $\begin{array}{c}\text { Drive } \\
\text { freq. }(\mathrm{Hz}) \\
\end{array}$ & $\tau_{I}$ & $\begin{array}{c}\text { MEG } \\
\text { data }\end{array}$ & $\begin{array}{c}\text { Full } \\
\text { model }\end{array}$ & $\begin{array}{c}\text { Full model, } \\
\text { no noise } \\
\end{array}$ & $\begin{array}{c}\text { Two-cell } \\
\text { model }\end{array}$ & $\begin{array}{c}\text { Behavior } \\
\text { classification }\end{array}$ \\
\hline 20 & $1 / 8$ & $\begin{array}{l}20 \\
40 \\
\end{array}$ & $\begin{array}{l}20 \\
40 \\
\end{array}$ & 20 & 20 & $\begin{array}{c}\text { Entrainment } \\
\text { Noise dependent }\end{array}$ \\
\hline 30 & $1 / 8$ & 30 & 30 & 30 & 30 & Entrainment \\
\hline 40 & $1 / 8$ & 40 & 40 & 40 & 40 & Entrainment \\
\hline 20 & $1 / 28$ & 20 & 20 & 20 & 20 & Entrainment \\
\hline 30 & $1 / 28$ & 30 & 30 & skip every 3rd & skip every $3 \mathrm{rd}$ & Noise dependent \\
\hline 40 & $1 / 28$ & $\begin{array}{l}20 \\
40 \\
\end{array}$ & $\begin{array}{l}20 \\
40 \\
\end{array}$ & 20 & 20 & $\begin{array}{c}\text { Alternate beat skipping } \\
\text { Noise dependent }\end{array}$ \\
\hline
\end{tabular}

4. Model phase space. The family of one-dimensional discontinuous discrete maps is based on the continuous ODE system (2.3)-(2.6) outlined above, and knowledge of the structures in the continuous phase space is critical for understanding map construction.

Note that the cells are distinguished only in the strength and timing of their synaptic input, so phase space for each, in the absence of any synaptic reset conditions, is identical. For each cell the $\theta$-nullcline is

$$
\theta_{ \pm}\left(g_{E}, g_{I}\right)= \pm \cos ^{-1}\left(\frac{1+\left(b+g_{E}-g_{I}\right)}{1-\left(b+g_{E}-g_{I}\right)}\right) .
$$

There are two fixed points of the continuous single-cell three-dimensional system at $\left(\theta_{\text {stab }}, 0,0\right)$ and $\left(\theta_{s d l}, 0,0\right)$, where $\theta_{s t a b}=\theta_{-}(0,0)$ and $\theta_{s d l}=\theta_{+}(0,0)$. The fixed point at $\left(\theta_{s t a b}, 0,0\right)$ is stable, while the fixed point at $\left(\theta_{s d l}, 0,0\right)$ is a saddle with a two-dimensional stable manifold and a one-dimensional unstable manifold that is exactly the $\theta$-axis. Figure 5 shows the phase space (defined on $S^{1} \times \mathbb{R}^{+} \times \mathbb{R}^{+}$) containing the $\theta$-nullcline, critical points, and several solutions to the system in the absence of reset in $g_{E}$ and $g_{I}$. The variable $g_{E}$ is plotted vertically, and the variable $g_{I}$ is plotted horizontally.

Figure 5 provides insight into the dynamic interactions between the $g_{E}$ and $g_{I}$ variables. In order for a cell to fire, it must be reset above the stable manifold of the saddle fixed point at $\left(\theta_{s d l}, 0,0\right)$, which lies above the $\theta$-nullcline. Positive change in $g_{E}$ can move solutions above the stable manifold and permit spiking. However, when inhibitory input is received and the $g_{I}$ value is increased, the solution moves into a region where much larger values of input to $g_{E}$ are required to boost the solution above the $\theta$-nullcline and further over the stable manifold.

When the system is restricted to the $g_{I}=0$ plane, the nullcline has two branches that cross the $g_{E}=0$ axis and meet when $g_{E}=-b$, as in Figure $6(\mathrm{~A})$. In the $g_{E}=0$ plane, there are

Copyright $\odot$ by SIAM. Unauthorized reproduction of this article is prohibited. 


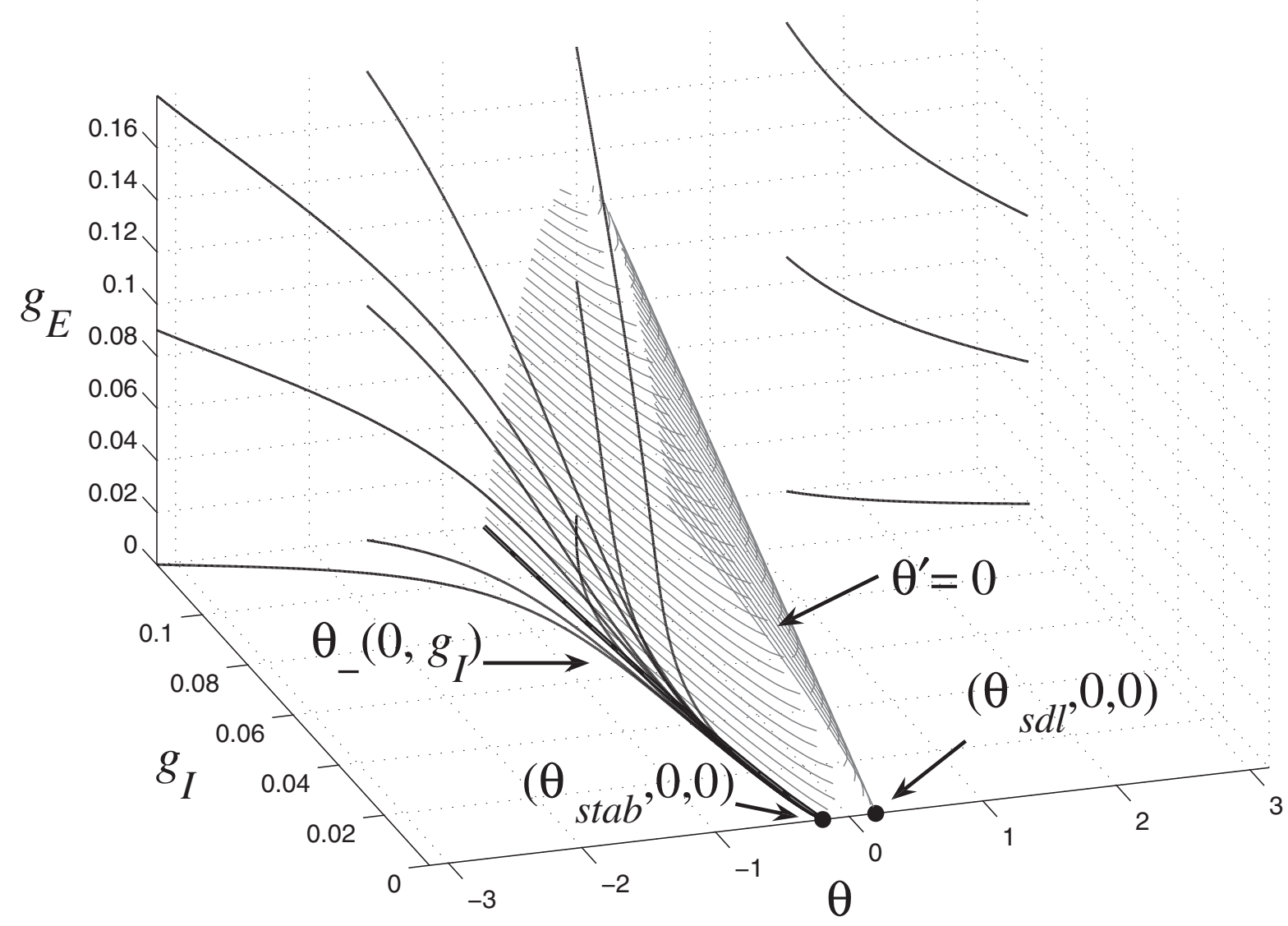

Figure 5. Flow for a single cell receiving excitatory and inhibitory input with parameters $\tau_{E}=1 / 2$ and $\tau_{I}=1 / 8$. Additional parameters are as in Table 1 . Several solutions and the $\theta$-nullcline are shown. The stable fixed point $\left(\theta_{\text {stab }}, 0,0\right)$, the saddle fixed point $\left(\theta_{\text {sdl }}, 0,0\right)$, and $\theta_{-}\left(0, g_{I}\right)$ are labeled.

two points on the $\theta$-nullcline for every value of $g_{I}$. Figure $6(\mathrm{~B})$ shows the intersection of the $\theta$-nullcline and the $g_{E}=0$ plane. The solution curves in Figures 5 and $6(\mathrm{~B})$ also indicate the presence of another important dynamic structure. A dominant feature of the phase space is the presence of a strongly attracting trajectory of solutions near the intersection of the stable branch of the $\theta$-nullcline and the $g_{E}=0$ plane, at $\theta_{-}\left(0, g_{I}\right)$. Such attracting trajectories have been studied previously in systems of ODEs and are referred to as rivers [9, 10]. A similar river has been previously described specifically in the context of the "theta" model [5]. In the system considered here, the river exists because the time scale for the inhibitory variable $g_{I}$ is significantly slower than that for the $g_{E}$ or $\theta$ variables. We later approximate values on the river by the corresponding value on this branch of the $\theta$-nullcline, at $\theta_{-}\left(0, g_{I}\right)$.

5. Discrete map development and computation. We develop a map $F:(0, T] \rightarrow(0, T]$ with input $\Delta t$, the length of time between the arrival of excitatory drive and the arrival of disynaptic inhibition to the E cell, where we assume that inhibition is triggered on that drive cycle. The output value, $F(\Delta t)$, is the time interval between drive input and inhibitory input to the E cell on the next cycle in which the I cell is triggered. Since excitatory input to the 
A

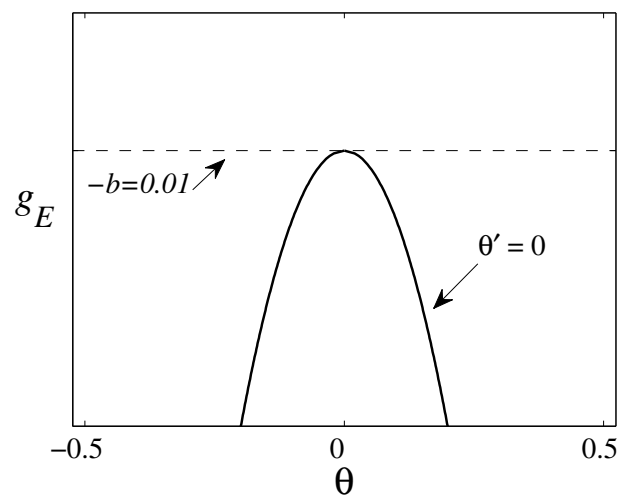

B

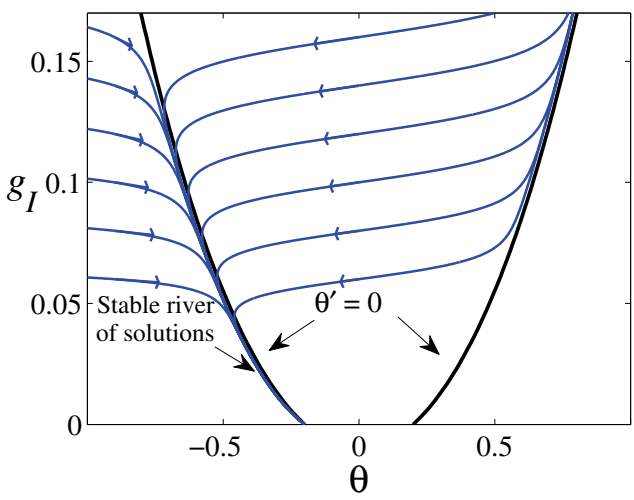

Figure 6. A: Phase plane for a single cell with zero inhibition $\left(g_{I}=0\right)$. The peak of the $\theta$-nullcline in this plane occurs at $\theta=0$ and $g_{E}=-b=-0.1$. B: Phase plane for a single cell with zero excitation $\left(g_{E}=0\right)$. Several solutions shown in blue are for $\tau_{I}=1 / 28$, and approach a stable river of solutions very near the stable branch of the $\theta$-nullcline.

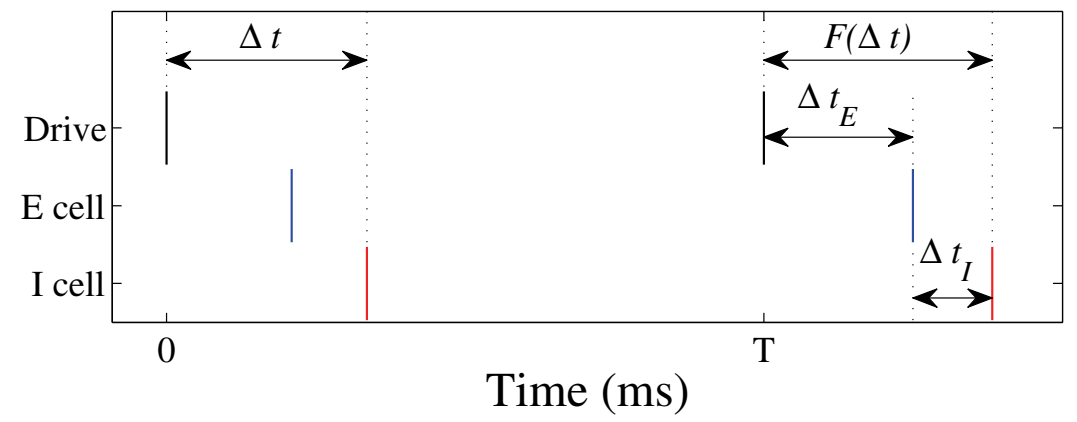

Figure 7. Raster plot showing two drive cycles and the relationship of $\Delta t$ and $F(\Delta t)$ to network firing times. $\Delta t_{E}$ and $\Delta t_{I}$ denote the contributions of the $E$ cell firing time and the I cell firing time to the total value of $\Delta t$. The map $F$ outputs the value of $\Delta t$ for the next drive cycle to which the network responds.

E cell is periodic with period $T$, and map output is measured as a distance in time that must be less than $T$, we can regard $F$ as a map from $S^{1}$ to $S^{1}$. However, we use interval notation in defining the map to emphasize dependence on the period $T$. Figure 7 shows the relation of $\Delta t$ and $F(\Delta t)$ to network firing times. In order to derive this map, we make the following critical assumption.

Assumption 5.1. We assume that, prior to excitatory input, solutions lie at the intersection of the stable branch of the $\theta$-nullcline and the $g_{E}=0$ plane, denoted $\theta_{-}\left(0, g_{I}\right)$.

This assumption is based on the strength of attraction to the stable river of solutions near the intersection of the $\theta$-nullcline and the $g_{E}=0$ plane, denoted $\theta_{-}\left(0, g_{I}\right)$ (as is shown in Figure 6(B)), as well as Assumption 2.4 that variable $g_{E}$ is negligible prior to excitatory input. The validity of this assumption will be discussed in section 8 below. In the discussion that follows we suppress the second $E$ and $I$ subscripts on $g_{E}$ and $g_{I}$ variables when statements apply generally to either recipient cell type.

Copyright (C) by SIAM. Unauthorized reproduction of this article is prohibited. 
Because inhibition may be such that the network fails to respond to one or more drive inputs, we construct $F$ using a family of curves, $\left\{F_{1}, F_{2}, \ldots, F_{n}\right\}$, in which each curve corresponds to a different number of missed drive responses, but no individual curve will necessarily cover the entire domain of $F$. We first consider the curve $F_{1}$ associated with the response to every drive input. By selecting an initial $\Delta t \in(0, T)$, it is implied that both excitatory and inhibitory response to the first drive input occurs prior to the second drive input. The larger the initial $\Delta t$, the later the inhibition arrives in that drive cycle, and the less inhibition has decayed by the time the next drive input arrives. It is possible for $\Delta t$ to be large enough that the $\mathrm{E}$ cell is sufficiently inhibited as to not spike in response to the second drive input, and thus not trigger inhibition. In this case we say that the map $F_{1}$ is undefined for that value of $\Delta t$. For such values, we consider $F_{2}$.

The second curve, $F_{2}$, is valid for values of $\Delta t$ for which the network (both the E cell and I cell) is silent in response to the second drive input but responds to a third input with time interval $F_{2}(\Delta t)$. The map $F_{3}$ and further $F_{i}$ are defined analogously, and the domains of the $F_{i}$ are mutually exclusive. Note that the output of any $F_{i}$ is in $(0, T]$ and hence is an input to one of the curves $F_{j}$. It is in this way that multiple curves can belong to the same map $F$ even though each curve is associated with additional information regarding skipped beats.

The map $F$ can be calculated using Assumption 5.1. Since we assume that solutions in both cell types are in the $g_{E}=0$ plane and on the stable branch of the $\theta$-nullcline prior to excitatory input, we need only know the level of inhibition in the cell at the time of excitatory input to locate the appropriate solution with which to numerically calculate spike times.

When an initial value of $\Delta t$ is selected, this determines the amount of time $T-\Delta t$ that the inhibitory variable in the $\mathrm{E}$ cell $\left(g_{I E}\right)$ has in which to decay before the next drive input arrives. Thus we can calculate the amount of inhibition present when the next input arrives, which we denote by $g_{I E}^{*}(\Delta t)$ to emphasize the dependence of this value on the initial selection of $\Delta t$. Recall here that $g_{I}$ is defined using simple exponential decay, so

$$
g_{I E}^{*}(\Delta t)=k_{I E} e^{-\tau_{I}(T-\Delta t)} .
$$

The relationship of time, $k_{I E}$, and $g_{I E}^{*}(\Delta t)$ are shown in Figure $8(\mathrm{~A})$. Now define $\theta_{-, E}^{*}=$ $\theta_{-}\left(0, g_{I E}^{*}(\Delta t)\right)$, which by Assumption 5.1 is the $\theta_{-}$value for the point along the stable branch of the $\theta$-nullcline where solutions reside prior to the second excitatory input to the $\mathrm{E}$ cell. This point is shown in the $g_{E}=0$ plane in Figure 8(B).

Recall from section 2 that we include a shift of $S$ radians in $\theta$ and a reset of the variable $g_{E, E}$ to $k_{E, E}$ after drive input to the E cell. Thus, just after excitatory input, E cell solutions have the coordinates $\theta=\theta_{-, E}^{*}+S, g_{E E}=k_{E E}$, and $g_{I E}=g_{I E}^{*}(\Delta t)$, as in (5.1). This initial condition is used to numerically calculate the length of time it takes the $\mathrm{E}$ cell to fire after excitatory drive input. The calculation of this escape time for the E cell, which we denote as $\Delta t_{E}$ (see Figure 7 ), is performed using a numeric ODE solver implemented in MATLAB (ode113). Note that $\Delta t_{E}$ may not exist if the reset solution is trapped beneath the $\theta$-nullcline surface (shown in Figure 5) and thus the $\mathrm{E}$ cell does not produce a spike. If the input value of $\Delta t$ is such that this occurs, then $F_{1}$ is not defined for this value of $\Delta t$.

Once $\Delta t_{E}$ is calculated, the length of time it takes the I cell to fire, $\Delta t_{I}$, must also be calculated (see Figure 7 for schematic). This calculation is identical to that for $\Delta t_{E}$, with the exception that both excitatory and inhibitory input sizes are different for the I cell (see 

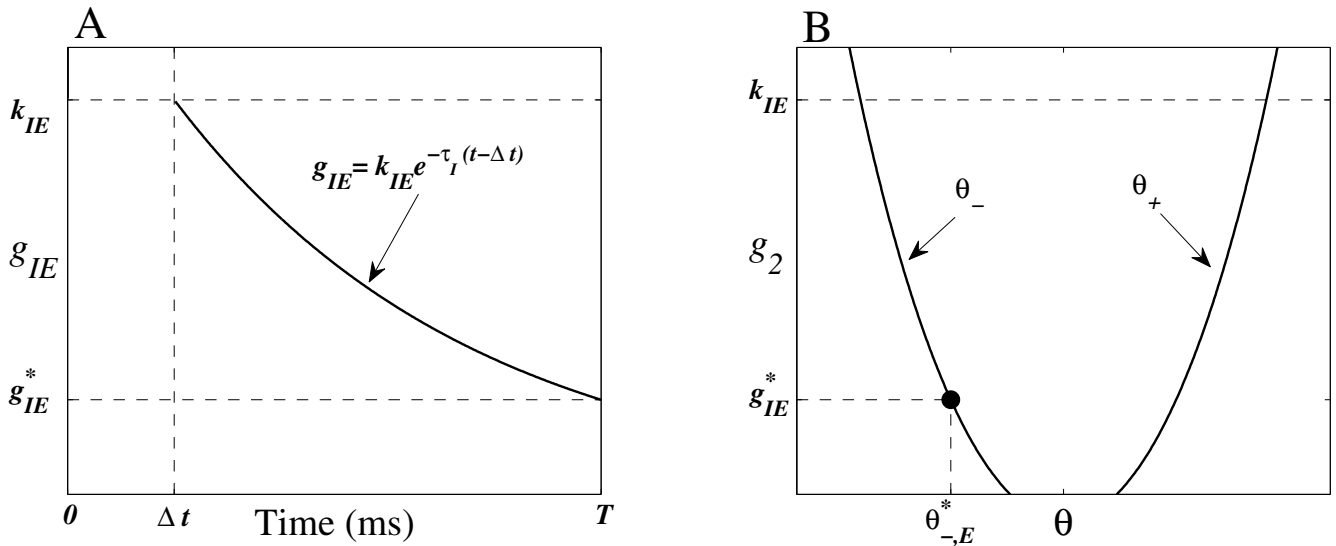

Figure 8. A: Evolution $g_{I E}$ after reset at $t=\Delta t$. Value at time $T$ is $g_{I E}^{*}$. B: Prior to excitatory input at time $T^{-}$, solutions in the $g_{E}=0$ plane are located at $\theta=\theta_{-, E}^{*}$ and $g_{I}=g_{I E}^{*}$, under the assumption that solutions lie directly on $\theta_{-}\left(0, g_{I}\right)$ (Assumption 5.1).

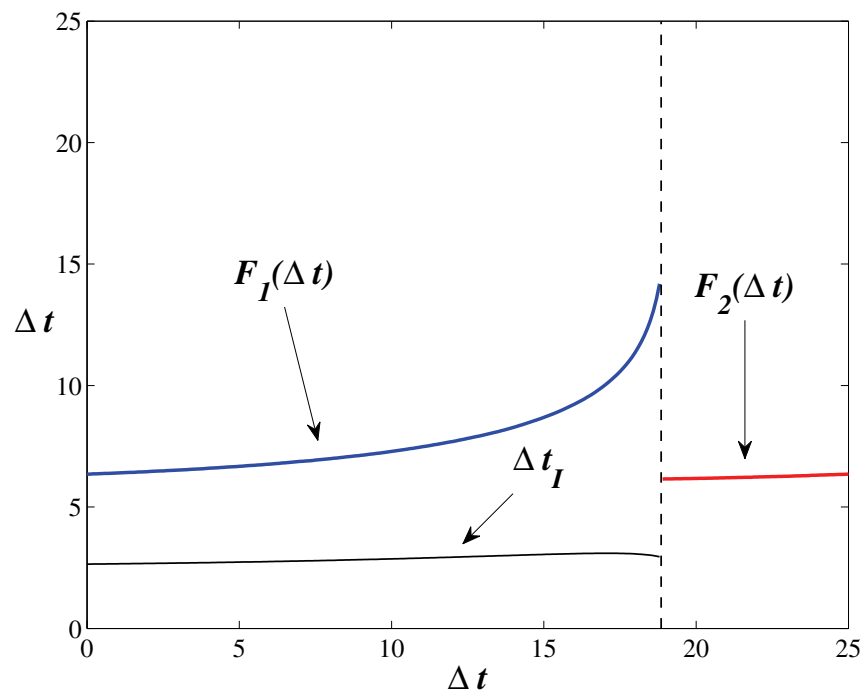

Figure 9. The curve $F_{1}$ for the case of $40 \mathrm{~Hz}$ drive with short inhibitory decay $\left(\tau_{I}=1 / 8\right)$. Note that $\Delta t_{I}$ remains fairly constant, and thus $\Delta t_{E}$ controls the shape of curve $F_{1}$. The dashed vertical line indicates the boundary of the domain of $F_{1}$.

Table 1), and the length of time for decay is longer by $\Delta t_{E}$. Thus we have

$$
g_{I I}^{*}(\Delta t)=k_{I I} e^{-\tau_{I}\left(T-\Delta t+\Delta t_{E}\right)} .
$$

To solve for $\Delta t_{I}$ we use the initial condition $\theta=\theta_{-, I}^{*}+S$, where $\theta_{-, I}^{*}=\theta_{-}\left(0, g_{I I}^{*}(\Delta t)\right)$, $g_{E} I=k_{E I}$, and $g_{I I}=g_{I I}^{*}(\Delta t)$, as in (5.2). Recall from Assumption 2.2 that parameters are such that if the $\mathrm{E}$ cell fires, the I cell will fire. Thus, if $\Delta t_{E}$ exists, so does $\Delta t_{I}$. We take $F(\Delta t)=\Delta t_{E}+\Delta t_{I}$. The resulting curve $F_{1}(\Delta t)$ is labeled in Figure 9 for short inhibitory decay $\left(\tau_{I}=1 / 8\right)$ and $T=25$.

Copyright (C) by SIAM. Unauthorized reproduction of this article is prohibited. 
For this parameter regime, $\Delta t_{E}$ controls the more interesting dynamics. By this we mean that $\Delta t_{I}$ remains fairly constant over the interval where the curve $F_{1}$ is defined and that $\Delta t_{E}$ controls the shape of the curve $F_{1}$. Figure 9 shows the contribution of $\Delta t_{I}$ to the curve $F_{1}$ for $T=25$ and $\tau_{I}=1 / 8$. The importance of $\Delta t_{E}$ stems from our assumption that parameters are such that any spike in the E cell guarantees a response in the I cell (Assumption 2.2). In order to meet this assumption, the excitatory input to the I cell is fairly strong, which causes more uniformity in the I cell spike time.

In the regions where $F_{1}$ is not defined, we must establish whether or not $F_{2}$ exists. This is done in the same manner except that, since no inhibitory input is received in response to the second drive pulse, both the $\mathrm{E}$ and I cells have an additional period of length $T$ for inhibition to decay. Thus, when we calculate the curve $F_{2}$, for the $\mathrm{E}$ cell we have

$$
g_{I E}^{*}(\Delta t)=k_{I E} e^{-\tau_{I}(2 T-\Delta t)},
$$

and for the I cell we have

$$
g_{I I}^{*}(\Delta t)=k_{E I} e^{-\tau_{I}\left(2 T-\Delta t+\Delta t_{E}\right)} .
$$

Because inhibitory variables have an additional period $T$ in which to decay, the map value for the lower end of the domain of $F_{2}$ is much smaller than for the upper end of the domain of curve $F_{1}$. This results in a jump discontinuity in the map $F$ taken as a whole, as can be seen in Figure 9. If the domains of $F_{1}$ and $F_{2}$ together are not sufficient to cover the interval on which the map $F$ is defined, $(0, T]$, then a third curve $F_{3}$ can be calculated analogously. For drive in the 20-40 $\mathrm{Hz}$ range and parameters considered here (see Table 1), in most cases $F_{1}$ and $F_{2}$ are sufficient to cover the map domain. However, for a limited range with drive near $40 \mathrm{~Hz}$ and longer inhibitory decay, $F_{2}$ and $F_{3}$ cover the domain. Because we generate this map computationally, we cannot know precisely the analytic domains for each curve. We do know, however, that since any solution corresponding to an initial condition reset from the stable river must either spike or be silent in response to excitatory input in finite time, the domains of the relevant $F_{n}$ will cover the entire interval $(0, T]$ and that the domains are mutually exclusive.

6. Maps $F(\Delta t)$ for different values of $\tau_{I}$. We now examine the numerically derived maps $F$ for fast $\left(\tau_{I}=1 / 8\right)$ and slow $\left(\tau_{I}=1 / 28\right)$ inhibitory decay, which correspond to the control and schizophrenic conditions in the previous work [28]. Parameters for these simulations are as in Table 1.

6.1. Maps $\boldsymbol{F}(\boldsymbol{\Delta} t)$ for $\tau_{I}=\mathbf{1} / 8$. Consider the case with $T=25$ and $\tau_{I}=1 / 8$. Figure 10(A) shows the map $F$ where $F_{1}$ and $F_{2}$ are labeled separately, calculated as described in section 5. Note that only $F_{1}$ and $F_{2}$ are necessary in order to define a map on the entire interval $(0, T]$.

This figure shows that there is a single stable fixed point of the map $F$ on the curve $F_{1}$. This implies that after a transient, the system will quickly settle into a pattern where the I cell fires at a fixed time $\Delta t \approx 6.8578 \mathrm{~ms}$ after each drive input. Because the fixed point occurs on the curve $F_{1}$ and not on the curve $F_{2}$, we also know that any beat skipping that may occur is transitory.

A similar structure is seen for the cases with $T=33.33 \mathrm{~ms}$ (30 Hz drive) and $T=50 \mathrm{~ms}$ (20 Hz drive), shown in Figures 10(B) and 10(C), respectively. For the case of $30 \mathrm{~Hz}$ drive, 

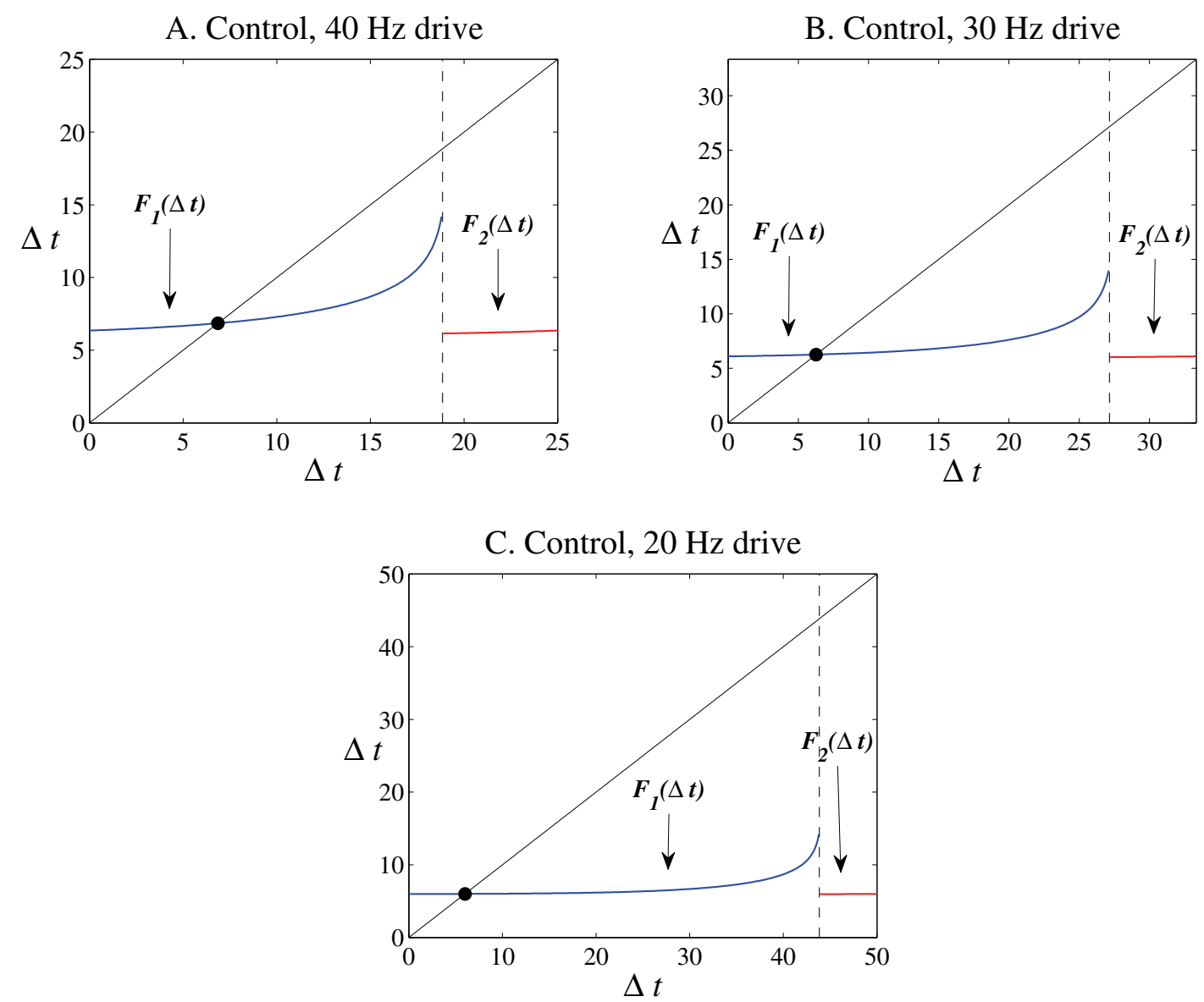

Figure 10. The map $F(\Delta t)$ generated computationally for control conditions $\left(\tau_{i}=1 / 8\right.$, parameters as in Table 1). The dashed vertical lines indicate the boundary between the domain of $F_{1}$ and the domain of $F_{2}$. A: $40 \mathrm{~Hz}$ drive, fixed point on $F_{1}$ located at $\Delta t \approx 6.8578$. B: $30 \mathrm{~Hz}$ drive, fixed point on $F_{1}$ located at $\Delta t \approx 6.2636$. C: $20 \mathrm{~Hz}$ drive, fixed point on $F_{1}$ located at $\Delta t \approx 6.0003$.

the discrete map settles into a fixed point at $\Delta t \approx 6.2636$. Similarly, for the case of $20 \mathrm{~Hz}$ drive, the map has a fixed point at $\Delta t \approx 6.0003$.

For all three of these cases, the fixed point is located on $F_{1}$, which indicates entrainment of the network to the frequency in a one-to-one manner. As the period increases, $\Delta t$ gets smaller, because a long period provides more time for inhibitory decay, which leads to faster spike times in both the E cell and the I cell.

6.2. Maps $F(\Delta t)$ for $\tau_{I}=1 / 28$. We now consider cases where inhibition decays much more slowly, with $\tau_{I}=1 / 28$. Under this condition, the curve $F_{2}$ gains a much larger domain in the interval $(0, T]$. The map for slow inhibitory decay with $40 \mathrm{~Hz}$ drive is shown in Figure 11(A). The curve $F_{1}$ does not exist for these parameters, and the fixed point is now deep within the $F_{2}$ curve at $\Delta t \approx 8.3798$. This corresponds to the beat skipping behavior in which the network fails to respond to alternate drive inputs in an extremely stable fashion.

Copyright (C) by SIAM. Unauthorized reproduction of this article is prohibited. 


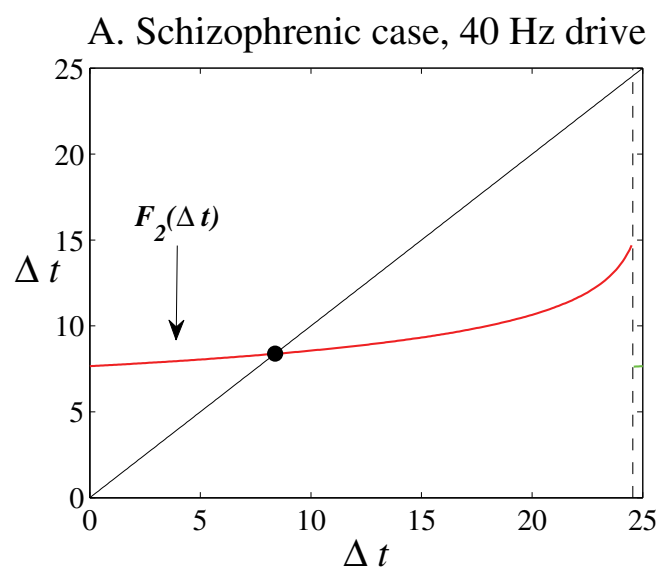

\section{B. Schizophrenic case, $30 \mathrm{~Hz}$ drive}

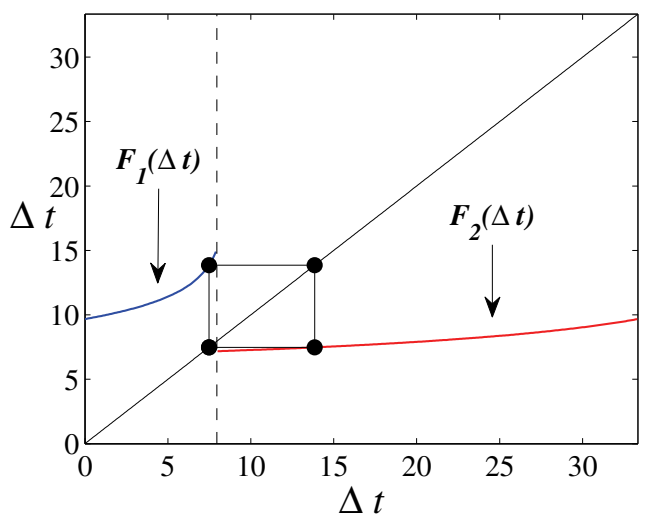

C. Schizophrenic case, $20 \mathrm{~Hz}$ drive

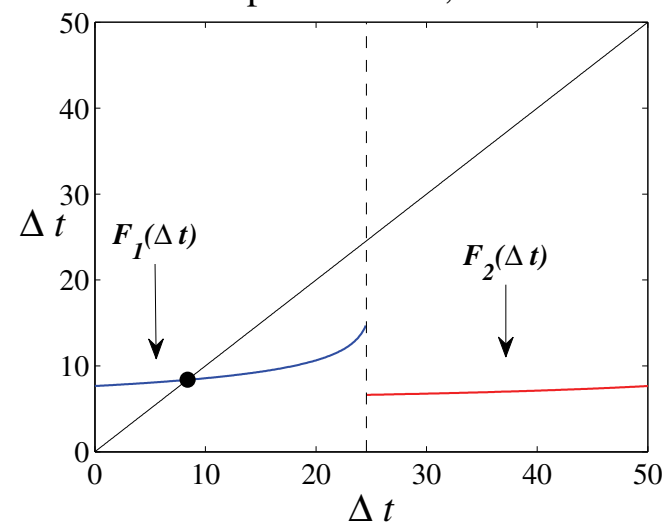

Figure 11. The map $F(\Delta t)$ generated computationally for schizophrenic conditions $\left(\tau_{i}=1 / 28\right.$, parameters as in Table 1). The dashed vertical lines indicate the boundary between the domains of $F_{1}, F_{2}$, and $F_{3}$. A: $40 \mathrm{~Hz}$ drive, fixed point on $F_{2}$ located at $\Delta t \approx 8.3798$. B: $30 \mathrm{~Hz}$ drive, no fixed point due to discontinuity. Stable 2-cycle indicated by rectangle. C: $20 \mathrm{~Hz}$ drive, fixed point on $F_{1}$ located at $\Delta t \approx 8.3798$.

Figure 11(B) shows the map $F$ with $30 \mathrm{~Hz}$ drive and $\tau_{I}=1 / 28$. There is no fixed point for the system because of the jump discontinuity between the curves $F_{1}$ and $F_{2}$. Instead of a fixed point, there is a stable 2-cycle with one point on $F_{1}$ and the other point on $F_{2}$, which corresponds to a network that fails to respond to every third drive input (behavior shown in Figure $4(\mathrm{~B}))$. Figure $11(\mathrm{C})$ shows the map $F(\Delta t)$ with $\tau_{I}=1 / 28$ and $20 \mathrm{~Hz}$ drive. The map has a fixed point on $F_{1}$ at $\Delta t \approx 8.3798$, which corresponds to entrainment to drive frequency.

7. Answers to motivating questions. Our previous study in larger networks left several questions unanswered regarding the schizophrenic case of slow inhibitory decay $\left(\tau_{I}=1 / 28\right)$, as outlined above in section 3. The map $F$ provides insight into all of these questions.

7.1. The "beat skipping" response to $40 \mathrm{~Hz}$ drive is robust. With $\tau_{I}=1 / 28$, the fixed point on $F_{2}$ explains the $20 \mathrm{~Hz}$ beat skipping component of the schizophrenic response to $40 \mathrm{~Hz}$ drive. The fixed point on $F_{2}$ implies that the network will fire at half of the drive 

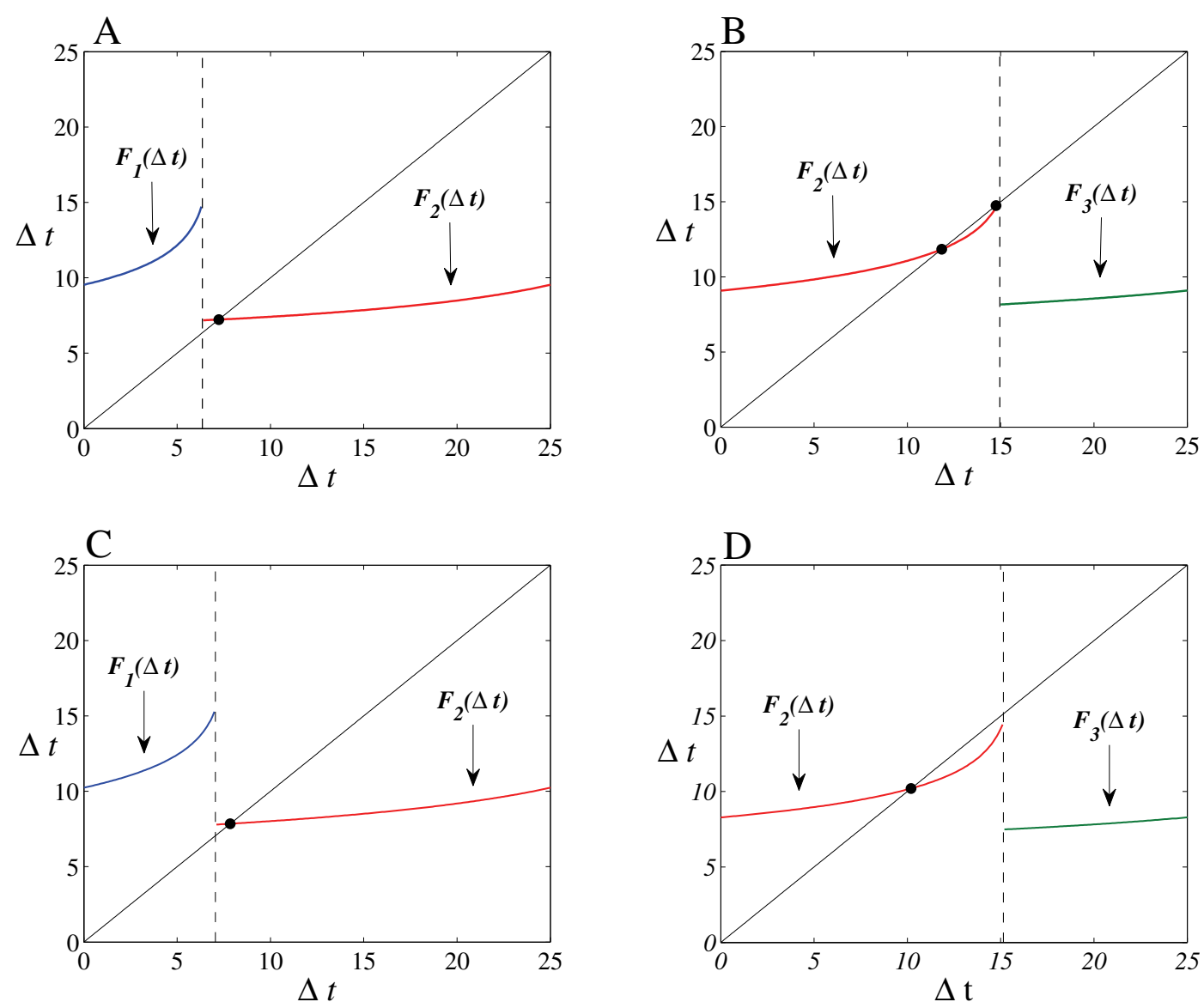

Figure 12. For $40 \mathrm{~Hz}$ drive with extended inhibition, a broad range of inhibitory parameters still produce a fixed point on $F_{2}$ and thus generate a $20 \mathrm{~Hz}$ response to $40 \mathrm{~Hz}$ drive. In all panels, only the parameter indicated in the caption is varied from original settings (see Table 1). A: Faster inhibitory decay, $\tau_{I}=1 / 21$. B: Slower inhibitory decay, $\tau_{I}=1 / 38$. Note the presence of two fixed points. The fixed point with a smaller value is stable. C: Weaker inhibition, $k_{I E}=0.115$. D: Stronger inhibition, $k_{I E}=0.21$.

frequency, responding in a stable fashion to alternate drive inputs (Figure 11(A)), producing a $20 \mathrm{~Hz}$ response to $40 \mathrm{~Hz}$ drive. The map $F$ provides a way to visualize the robustness of this mechanism. In Figure 12, maps near the extreme values of both inhibitory decay and inhibitory strength for which beat skipping occurs are shown. In Figure 12(A), the map with $\tau_{I}=1 / 21$ is shown, which is somewhat faster than in the original simulations but still produces the fixed point on $F_{2}$. In Figure $12(\mathrm{~B})$, we take $\tau_{I}=1 / 38$, which is a much slower decay than that used for the computations above but still produces a fixed point on the $F_{2}$ curve, and thus beat skipping persists. Similarly, values $k_{I E}=0.115$ and 0.21 , which represent the strength of inhibition provided to the excitatory cell, are shown in Figures 12(C) and 12(D). Both have a fixed point on $F_{2}$ and produce the same overall network behavior of skipping alternate drive inputs. This is in contrast to the transitional behavior, where network entrainment is extremely sensitive to even very small parameter changes.

Copyright (C) by SIAM. Unauthorized reproduction of this article is prohibited. 
7.2. Less-frequent beat skipping patterns are generated by the discontinuity in $F$ and are dynamically distinct from the alternate beat skipping in the case of $40 \mathrm{~Hz}$ drive. One motivation for study of this map was that, when examining the computational model for the schizophrenic case with $30 \mathrm{~Hz}$ drive for various parameters, we observed model behavior of skipping beats, but less frequently than alternate beats. There was some concern that this might be a problem with the computational model: if such alternate behaviors were robust and the hypothesis about extended inhibition was correct, why was only alternate beat skipping indicated in the experimental results?

From Figures 11(B) and 11(C) we can discern that, under the slow inhibition condition $\left(\tau_{I}=1 / 28\right)$, the map $F$ transitions from locked response to every drive pulse to beat skipping somewhere between $T=50 \mathrm{~ms}$ and $T=25 \mathrm{~ms}$ as the period $T$ is reduced. The discontinuity in $F$ leads to a rich transitional behavior as the period $T$ is varied. Because of the discontinuity, there is a range of values in $T$ such that the map $F$ does not have a fixed point, such as the case above when $T=33.33$, shown in Figure 11(B). Instead of a fixed point, the map $F$ contains a limit cycle, indicated by the black box connecting a point on $F_{1}$ to a point on $F_{2}$, which corresponds to the network skipping every third drive input. As long as the map $F$ has no fixed points, the network will skip beats, but less frequently than alternate beat skipping. All simulations indicate that the behavior reflects a stable limit cycle in that the pattern of beat skipping appears very regular, possibly after an initial transient.

As $T$ is increased further and nears the regime where the network responds to every drive pulse, the network responds more times to the drive before beat skipping. For $T=39$ the map $F$ is drawn in Figure 13(A). Note how close the curve $F_{1}$ is to the diagonal at $\Delta t=\Delta t$. This narrow region between the diagonal line and the curve $F_{1}$ produces a long limit cycle before the solution escapes to the $F_{2}$ curve and skips an input pulse.

The network enters the locking regime via a saddle node bifurcation as $T$ is increased. The resulting two fixed points can be seen in Figure 14 for $T=40$, but the unstable fixed point is present only in a very small range of parameter space. No chaotic behavior was observed in simulations for this transitional regime, though it is likely present, particularly near the saddle node bifurcation on $F_{1}$.

These less frequent beat skipping patterns are generated by the lack of a fixed point for the map $F$, due to the discontinuity between curves $F_{1}$ and $F_{2}$, and are extremely sensitive to even very slight parameter changes. This mechanism is in stark contrast to the fixed point on $F_{2}$ that generates the alternate beat skipping mechanism seen for $40 \mathrm{~Hz}$ drive (see section 7.1), which exists for a broad range of inhibitory parameters. The sensitive nature of this transitional regime indicates that less-frequent skipping behavior, if present experimentally, would be rendered invisible by the process of data processing, in which there is averaging across subjects who would likely have highly variable skipping patterns due to the sensitivity of this transitional regime.

7.3. Presence of excitatory background noise favors $30 \mathrm{~Hz}$ entrainment due to sensitivity of network behavior in the transitional regime. In previous work with larger networks, we noticed that the presence of excitatory background noise strongly favored one-to-one entrainment for the schizophrenic case with $30 \mathrm{~Hz}$ drive. Although noise is not included in the reduced system used to generate the map $F$, the map structure provides insight into this sensitivity. For the $30 \mathrm{~Hz}$ case with $\tau_{I}=1 / 28$, the map $F$ has no fixed point due to the 

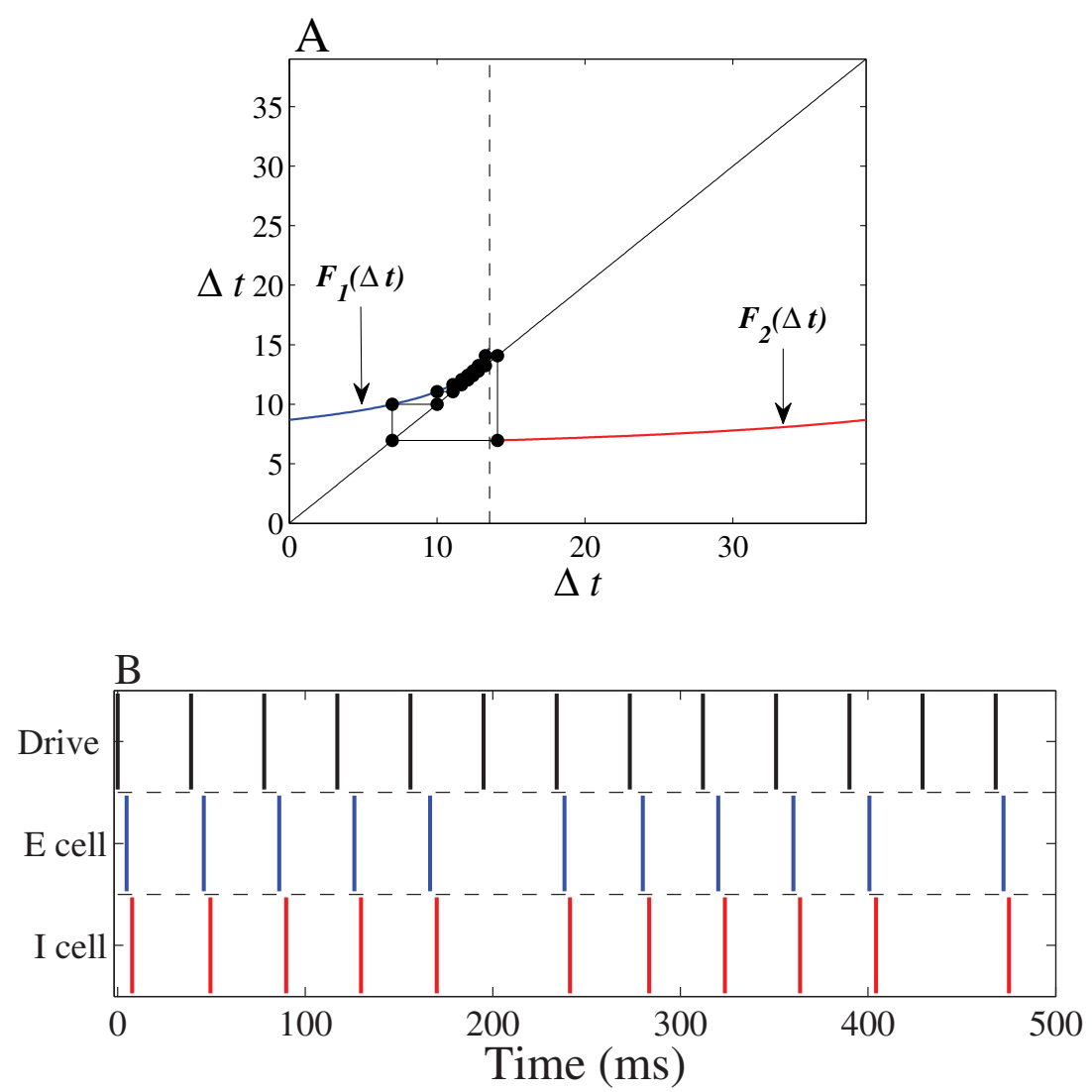

Figure 13. The discrete map $F(\Delta t)$ for long inhibitory decay and $T=39 \mathrm{~ms}$ displays infrequent beat skipping. A: The map $F(\Delta t)$ for $T=39 \mathrm{~ms}$ with slow inhibitory decay $\left(\tau_{I}=1 / 28\right)$. Curves $F_{1}$ and $F_{2}$ are indicated. The dashed vertical line indicates the boundary between the domain of $F_{1}$ and the domain of $F_{2}$. There is no fixed point on either $F_{1}$ or $F_{2}$, though there is a nine-point stable limit cycle indicated by the cobwebbing linking points on the cycle. B: Raster plot for two-cell model with $T=39$ ms and no noise input. Note that skipping is more frequent in the raster than would be indicated by the limit cycle (skips every 6th input, analogous to a five-point limit cycle). This discrepancy is due to an effect of Assumption 5.1. See section 8 for further discussion.

discontinuity between $F_{1}$ and $F_{2}$ and is such that there is a stable 2-cycle. This corresponds to a network that fails to respond to every third pulse of the drive, as shown in Figure 15(A) for the two-cell system, with the coordinate values of the map points labeled. Figure 15(B) shows how the map values $a$ and $b$ correspond to the timing of the two-cell network response to $30 \mathrm{~Hz}$ drive. This behavior is identical to that seen without noise in the full system studied previously (see Figure 14 in [28]). Because the transitional regime where this case falls is very sensitive, small amounts of additional excitation can have large effects. For the larger model, excitatory background noise breaks this skipping into a less predictable pattern, with the network responding to almost all drive cycles, though somewhat inconsistently (see Figure 15B in [28]). Response to all drive cycles corresponds to $30 \mathrm{~Hz}$ entrainment.

8. Validity of the assumption that solutions lie on a stable branch of the $\theta$-nullcline prior to excitatory input. Recall that, in the construction of the map $F$, we assume that 


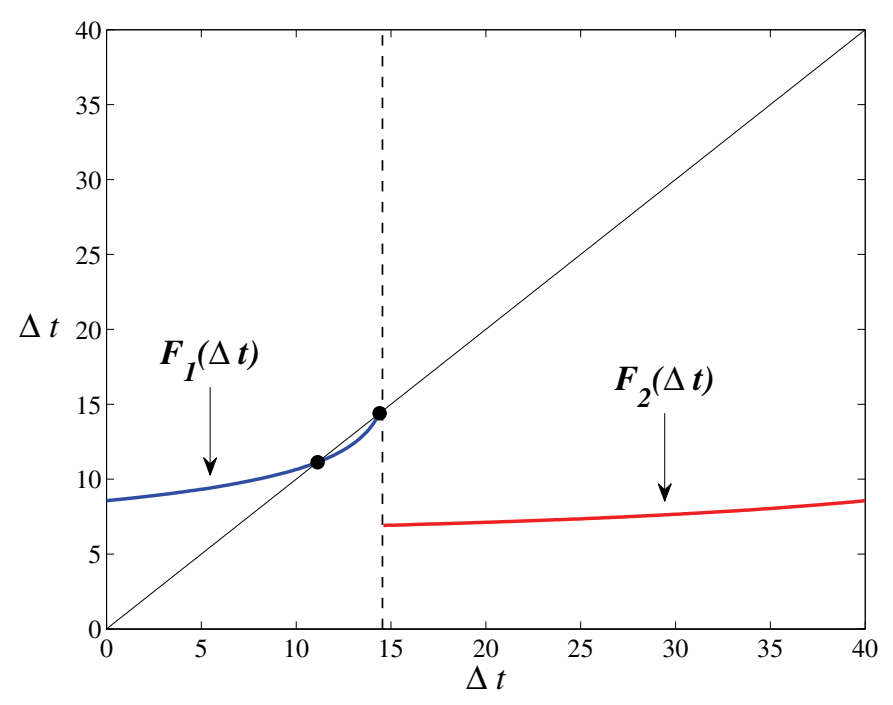

Figure 14. The map $F(\Delta t)$ for $\tau_{I}=1 / 28$ and $T=40 \mathrm{~ms}$ with curves $F_{1}$ and $F_{2}$ indicated. The dashed vertical line indicates the boundary between the domain of $F_{1}$ and the domain of $F_{2}$. Two fixed points due to a saddle node bifurcation are indicated by black dots.

in both cells, solutions lie on the intersection of the stable branch of the $\theta$-nullcline and the $g_{E}=0$ plane prior to excitatory input (Assumption 5.1). This assumption is possible because of the presence of a strongly attracting river of solutions in very close proximity to $\theta_{-}\left(0, g_{I}\right)$ (see section 4, Figures 5 and 6(B)), as well as because of Assumption 2.4 that $\tau_{E}$ and $T$ are such that the value of $g_{E}$ is negligible prior to excitatory input in both cells. While Assumption 5.1 is largely reasonable and produces a map that describes the most important qualitative network behaviors, it is not exact and does produce some minor discrepancies between the behavior of the ODE system (2.3)-(2.6) and map behaviors.

For the schizophrenic case, both the map and the dynamically defined system (2.3)-(2.6) transition out of locked one-to-one entrainment at $T=41$ and into beat skipping at $T=32$ as the period is decreased, to a resolution of $1 \mathrm{~ms}$ in $T$. These values also agree with the transitional values of the full computational model when noise is removed (data not shown; see [28]). Although there is excellent qualitative agreement for these important behaviors, Assumption 5.1 does cause slight overestimates of the value of $\theta$ prior to excitatory input. This overestimate encourages slightly more spiking in the transitional regime and predicts slightly smaller fixed point values of $\Delta t$ than are present in the continuous two-cell dynamical system. The effects of this discrepancy are most obvious in the sensitive transitional regime, particularly near the saddle node bifurcation, as in Figure 13, with slow decay $\left(\tau_{I}=1 / 28\right)$ and $T=39$. Note that the map produces a nine-point limit cycle (Figure $13(\mathrm{~A})$ ), which predicts that the system will skip every tenth drive input, but the raster plot produced dynamically from the ODE system (2.3)-(2.6) actually skips every sixth input (Figure 13(B)).

Assumption 5.1 also has implications for consideration of the map $F$ in response to other frequencies. In particular, for very fast frequencies, where excitatory inputs arrive in quick succession, there may not be sufficient time for solutions to approach the stable branch of 

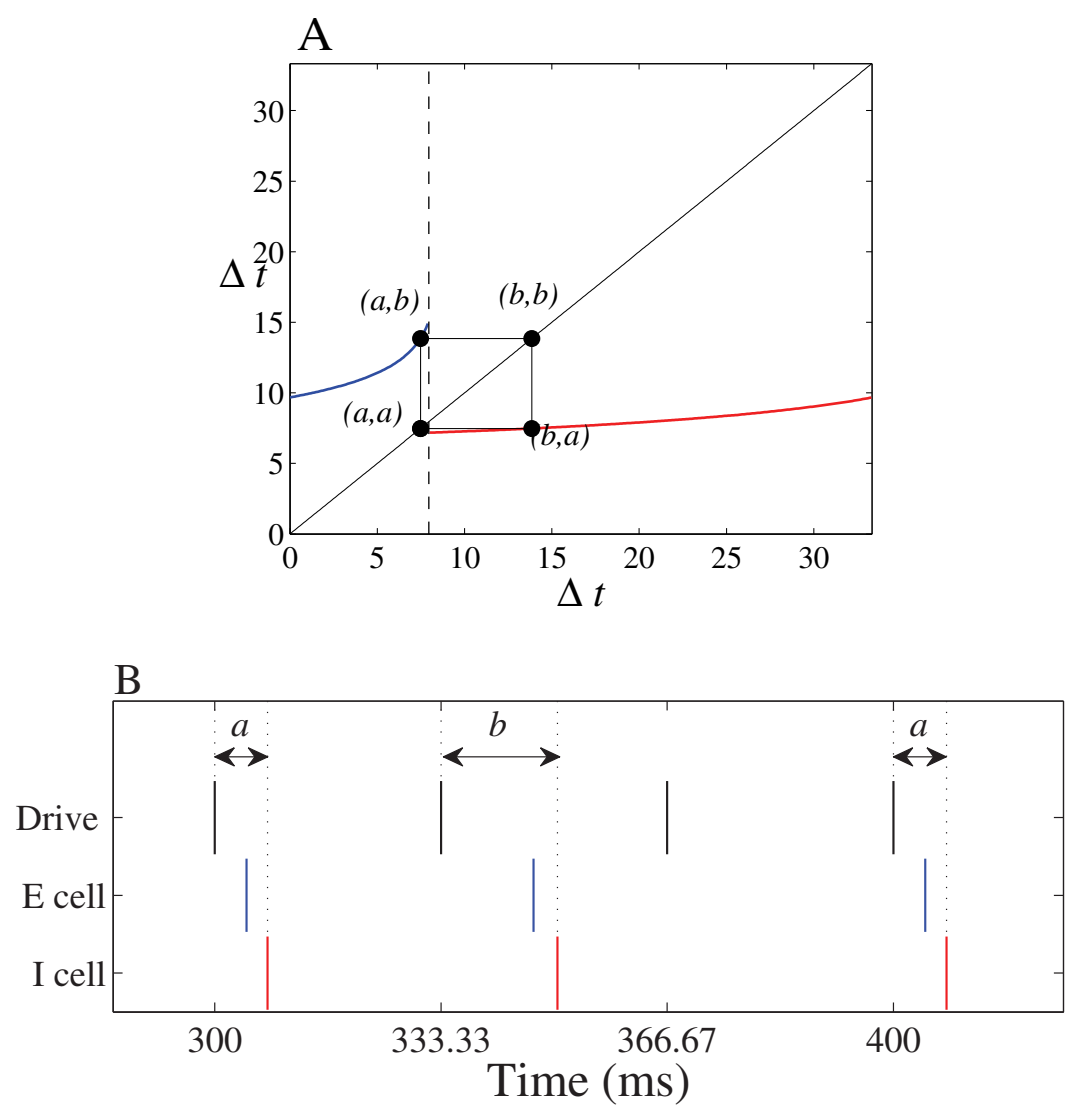

Figure 15. $30 \mathrm{~Hz}$ case for slow decay $\left(\tau_{I}=1 / 28\right)$, schizophrenic case. A: The map $F(\Delta t)$, with the limiting two-cycle indicated by the box; $a$ and $b$ are the two values taken by the map $F$ on this limit cycle. B: Portion of raster plot for the $30 \mathrm{~Hz}$ case, after the system has closely approached the limiting 2-cycle, showing how values $a$ and $b$ correspond to model entrainment behavior.

the $\theta$-nullcline. However, if this is the case, it is probable that Assumption 2.4 is violated. In addition, parameter regimes with faster inhibitory decay will cause this assumption to generate a greater overestimate in $\theta$, and thus will reduce agreement between the dynamical system and the map $F$. Conversely, if inhibition is slowed further, the assumption will become even more accurate, and discrepancies between the map $F$ and the ODE system behavior will be minimized.

9. Conclusions and future directions. The map $F$ provides insight into the dynamic impact of extended inhibition in E-I neural networks driven in the gamma/beta range. As discussed in the introduction, this work was originally inspired by study of altered auditory entrainment in schizophrenia [28]. That previous work examined whether extended inhibition caused by reduction in an available transporter (GAT-1) for the inhibitory neural transmitter GABA [21] might be sufficient to cause a preference for $20 \mathrm{~Hz}$ entrainment to gamma and beta range auditory click trains in schizophrenic subjects.

Work using the discrete map $F$ addresses several questions that could not be answered in our previous computational framework. In particular, we have now established the following: 
1. The "beat skipping" response to $40 \mathrm{~Hz}$ drive with slow inhibitory decay is extremely robust. The mechanism that generates this behavior (a fixed point on curve $F_{2}$ for $T=25$ ) is present for a wide range of parameters, which increases the likelihood that such a mechanism underlies network behavior in a living brain, where true biophysical parameters are likely to vary substantially, both within and between subjects.

2. Less-frequent skipping patterns in the model (e.g., skipping every third drive input), which were not observed experimentally, are dynamically distinct from the alternate beat skipping response. Such patterns are extremely sensitive to even slight parameter alterations, and would thus be expected to vary markedly from subject to subject, rendering such behavior invisible in averaged data.

3. With $30 \mathrm{~Hz}$ drive and slow inhibitory decay, the simplified two-cell network is in the transitional regime where the network fails to respond to every third drive input. This corresponds exactly with the behavior seen in the previous larger network when noise is removed (see Table 2). We see from the map $F$ that in this transitional regime, any skipping behavior will be extremely fragile and sensitive to outside excitation, which explains the particular dependence of the schizophrenic case with $30 \mathrm{~Hz}$ drive on excitatory background noise for pure $30 \mathrm{~Hz}$ entrainment in our previous work.

The previous model contained several features that have been eliminated in this reduced two-cell system, namely excitatory background noise, weak E-to-E connectivity, and weak drive to the I cells. Despite these reductions, the map $F$ clearly illustrates the dynamics underlying the most important entrainment behaviors. The presence of a fixed point on the curve $F_{1}$ under control parameters clearly illuminates the mechanism by which the full computational model entrains to 30 and $40 \mathrm{~Hz}$ drive, even in the presence of noise. For these cases, the fixed point on $F_{1}$ is extremely stable (see Figures 10(A) and 10(B)). Because these fixed points are deep within the $F_{1}$ curve, and located where the slope of $F_{1}$ is only slightly positive, attraction to the fixed point is very rapid, and one-to-one entrainment begins almost immediately. Perturbations due to noise or moderate network parameter modifications are unlikely to disrupt such a stable entrainment pattern. For the control case of $20 \mathrm{~Hz}$ drive, in which the full model shows a mixed $20 \mathrm{~Hz}$ and $40 \mathrm{~Hz}$ response, the fixed point on $F_{1}$ (Figure $10(\mathrm{C})$ ) explains the $20 \mathrm{~Hz}$ response, but does not explain the presence of $40 \mathrm{~Hz}$ activity. Here, the inclusion of excitatory background noise is essential to generate the $40 \mathrm{~Hz}$ component, and was examined in previous work, but is not included in this simplified case. When noise is present in the full model, inhibition triggered by the $20 \mathrm{~Hz}$ excitatory drive gates the excitatory background noise, generating a $40 \mathrm{~Hz}$ component to the signal.

Although we considered a specific parameter regime here, the same general analysis can be applied to other parameter regimes, provided that the initial assumptions in section 2 are met. Similar techniques might prove useful throughout study of the nervous system, where rhythmically driven networks are ubiquitous, though not necessarily with so clock-like a drive as the one considered here. Indeed, map families like the one developed here may prove useful for study of forced circuits in applications beyond neuroscience. Entrainment behavior seen in this small periodically driven network is similar to the $n: m$ locking described for weakly coupled oscillators [13], which has proven quite amenable to analytic study. However, to our knowledge, no analogous analytic framework has been developed for small forced circuits. Such an analytical framework may prove to be both accessible and powerful.

Copyright (c) by SIAM. Unauthorized reproduction of this article is prohibited. 
Acknowledgments. The authors wish to thank collaborators S. Stufflebeam and P. Siekmeier for data and modeling that helped inspire this work, as well as T. J. Kaper and D. A. Lewis for helpful discussions.

\section{REFERENCES}

[1] I. Belykh And A. Shilnikov, When weak inhibition synchronizes strongly desynchronizing networks of bursting neurons, Phys. Rev. Lett., 101 (2008), paper 078102.

[2] O. Bertrand and C. Tallon-Baudry, Oscillatory gamma activity in humans: A possible role for object representation, Int. J. Psychophysiol., 38 (2000), pp. 211-223.

[3] A. Bibbig, R. D. Traub, And M. A. Whittington, Long-range synchronization of gamma and beta oscillations and the plasticity of excitatory and inhibitory synapses: A network model, J. Neurophysiol., 88 (2002), pp. 1634-1654.

[4] C. Börgers, S. Epstein, And N. Kopell, Background gamma rhythmicity and attention in cortical local circuits: A computational study, Proc. Natl. Acad. Sci. USA, 102 (2005), pp. 7002-7007.

[5] C. Börgers And N. Kopell, Synchronization in networks of excitatory and inhibitory neurons with sparse, random connectivity, Neural Comput., 15 (2003), pp. 509-538.

[6] C. Börgers AND N. Kopell, Effects of noisy drive on rhythms in networks of excitatory and inhibitory neurons, Neural Comput., 17 (2005), pp. 557-608.

[7] C. Börgers and N. Kopell, Gamma oscillations and stimulus selection, Neural Comput., 20 (2008), pp. 383-414.

[8] M. O. Cunningham, M. A. Whittington, A. Bibbig, A. Roopun, F. E. N. LeBeau, A. Vogt, H. Monyer, E. H. Buhl, And R. D. Traub, A role for fast rhythmic bursting neurons in cortical gamma oscillations in vitro, Proc. Natl. Acad. Sci. USA, 101 (2004), pp. 7152-7157.

[9] F. Diener, Propriétés asymptotiques des fleuves, C. R. Acad. Sci. Paris Sér. I Math., 302 (1986), pp. $55-58$.

[10] M. Diener, Détermination et existence des fleuves en dimension deux, C. R. Acad. Sci. Paris Sér. I Math., 301 (1985), pp. 899-902.

[11] B. Doiron, B. Lindner, A. Longtin, L. Maler, and J. Bastian, Oscillatory activity in electrosensory neurons increases with the spatial correlation of the stochastic input stimulus, Phys. Rev. Lett., 93 (2004), paper 048101.

[12] A. K. Engel And W. Singer, Temporal binding and the neural correlates of sensory awareness, Trends Cogn. Sci., 5 (2001), pp. 16-25.

[13] G. B. Ermentrout, $n: m$ phase-locking of weakly coupled oscillators, J. Math. Biol., 12 (1981), pp. 327-342.

[14] G. B. Ermentrout And N. Kopell, Parabolic bursting in an excitable system coupled with a slow oscillation, SIAM J. Appl. Math., 46 (1986), pp. 233-253.

[15] M. Galarreta And S. Hestrin, Properties of GABAa receptors underlying inhibitory synaptic currents in neocortical pyramidal neurons, J. Neurosci., 17 (1997), pp. 7220-7227.

[16] J. Guckenheimer And P. Holmes, Nonlinear Oscillations, Dynamical Systems, and Bifurcations of Vector Fields, Springer-Verlag, New York, 1983.

[17] C. Haenschel, T. Baldeweg, R. J. Croft, M. Whittington, and J. Gruzelier, Gamma and beta frequency oscillations in response to novel auditory stimuli: A comparison of human electroencephalogram (EEG) data with in vitro models, Proc. Natl. Acad. Sci. USA, 97 (2000), pp. 7645-7650.

[18] M. Hausser AND A. Roth, Estimating the time course of the excitatory synaptic conductance in neocortical pyramidal cells using a novel voltage jump method, J. Neurosci., 17 (1997), pp. 7606-7625.

[19] B. J. Hefti And P. H. Smith, Distribution and kinetic properties of GABAergic inputs to layer $V$ pyramidal cells in rat auditory cortex, J. Assoc. Res. Otolaryngol., 4 (2003), pp. 106-121.

[20] J. S. Kwon, B. F. O’Donnel, G. V. Wallenstein, R. W. Greene, Y. Hirayasu, P. G. Nestor, M. E. Hasselmo, G. F. Potts, M. E. Shenton, and R. W. McCarley, Gamma frequency-range abnormalities to auditory stimulation in schizophrenia, Arch. Gen. Psych., 56 (1999), pp. 1001-1005.

[21] D. A. Lewis, T. Hashimoto, And D. W. Volk, Cortical inhibitory interneurons and schizophrenia, Nature Rev. Neurosci., 6 (2005), pp. 312-324.

Copyright (c) by SIAM. Unauthorized reproduction of this article is prohibited. 
[22] G. A. Light, J. L. Hsu, M. H. Hsieh, K. Meyer-Gomes, J. Sprock, N. R. Swerdlow, And D. L. BRAFF, Gamma band oscillations reveal neural network cortical coherence dysfunction in schizophrenia patients, Biolog. Psych., 60 (2006), pp. 1231-1240.

[23] D. Marinazzo, H. J. Kappen, and S. C. Gielen, Input-driven oscillations in networks with excitatory and inhibitory neurons with dynamic synapses, Neural Comput., 19 (2007), pp. 1739-1765.

[24] L. S. Overstreet And G. L. Westbrook, Synapse density regulates independence at unitary inhibitory synapses, J. Neurosci., 23 (2003), pp. 2618-2626.

[25] Q. PAuluis, S. N. BAKer, AND E. Olivier, Emergent oscillations in a realistic network: The role of inhibition and the effect of the spatiotemporal distribution of the input, J. Comput. Neurosci., 6 (1999), pp. 289-310.

[26] P. A. SAlin AND D. A. PRINCE, Electrophysiological mapping of GABAa receptor-mediated inhibition in adult rat somatosensory cortex, J. Neurophysiol., 75 (1996), pp. 1589-1600.

[27] P. A. SAlin AND D. A. PRInce, Spontaneous GABAa receptor-mediated inhibitory currents in adult rat somatosensory cortex, J. Neurophysiol., 75 (1996), pp. 1573-1588.

[28] D. Vierling-Claassen, P. Siekmeier, S. Stufflebeam, and N. Kopell, Modeling GABA alterations in schizophrenia: A link between impaired inhibition and altered gamma and beta range auditory entrainment, J. Neurophysiol., 99 (2008), pp. 2656-2671.

[29] M. A. Whittington, R. D. Traub, and J. G. Jefferys, Synchronized oscillations in interneuron networks driven by metabotropic glutamate receptor activation, Nature, 373 (1995), pp. 612-615.

[30] F. M. Zhou AND J. J. Hablitz, AMPA receptor-mediated epscs in rat neocortical layer II/III interneurons have rapid kinetics, Brain Res., 780 (1998), pp. 166-169.

Copyright $@$ by SIAM. Unauthorized reproduction of this article is prohibited. 